\title{
Renormalization group analysis on emergence of higher rank symmetry and higher moment conservation
}

\author{
Hongchao $\mathrm{Li}^{1,2, *}$ and Peng $\mathrm{Ye}^{1, \dagger}$ \\ ${ }^{1}$ School of Physics and State Key Laboratory of Optoelectronic Materials and Technologies, Sun Yat-sen University, \\ Guangzhou 510275, China \\ ${ }^{2}$ Department of Modern Physics, University of Science and Technology of China, Hefei 230026, China
}

(Received 24 April 2021; revised 25 November 2021; accepted 30 November 2021; published 13 December 2021)

\begin{abstract}
Higher rank symmetry and higher moment conservation have been drawn considerable attention from, e.g., subdiffusive transport to fracton topological order. In this paper, we perform a one-loop renormalization group (RG) analysis and show how these phenomena emerge at low energies. We consider a $d$-dimensional model of interacting bosons of $d$ components. At higher rank-symmetric points with conserved angular moments, the $a$ th bosons have kinetic energy only along the $\hat{x}^{a}$ direction. Therefore, the symmetric points look highly anisotropic and fine-tuned. By studying RG in a wide vicinity of the symmetric points, we find that symmetry-disallowed kinetic terms tend to be irrelevant within the perturbative regime, which potentially leads to emergent higher rank symmetry and higher moment conservation at the deep infrared limit. While nonperturbative analysis is called for in the future, by regarding higher rank symmetry as an emergent phenomenon, the RG analysis presented in this paper holds alternative promise for realizing higher rank symmetry and higher moment conservation in experimentally achievable systems.
\end{abstract}

DOI: 10.1103/PhysRevResearch.3.043176

\section{INTRODUCTION}

The celebrated Noether theorem relates a conservation law to an underlying continuous symmetry. For example, in a U(1)-symmetric Hamiltonian of bosons, bosonic operator $\hat{\Phi}(\mathbf{x})$ is changed to $e^{i \theta} \hat{\Phi}(\mathbf{x})$ under symmetry transformations where the real parameter $\theta$ does not depend on coordinate $\mathbf{x}=\left(x^{1}, x^{2}, \ldots, x^{d}\right)$ in $d$-dimensional space. By means of Noether's theorem, one can show that the total boson number, i.e., $\int d^{d} x \rho(\mathbf{x})$, is a conserved quantity, where particle number density $\rho(\mathbf{x})=\hat{\Phi}^{\dagger}(\mathbf{x}) \hat{\Phi}(\mathbf{x})$. Apparently, $\rho(\mathbf{x})$ is just the zeroth order of conventional multipole expansions

$$
\rho, \quad \rho \mathbf{x}, \quad \rho x^{a} x^{b}, \quad \cdots
$$

in a standard electromagnetism textbook [1]. In a particlenumber-conserving system, higher moment conservation, e.g., conservation of dipoles and quadrupoles, is in principle allowed. Furthermore, if the density is vectorlike with multiple components, denoted as $\rho=\left(\rho^{1}, \rho^{2}, \ldots, \rho^{d}\right)$, then we can

\footnotetext{
*Present address: Department of Physics, University of Tokyo, 7-3-1 Hongo, Bunkyo-ku, Tokyo 113-0033, Japan.

†yepeng5@mail.sysu.edu.cn

Published by the American Physical Society under the terms of the Creative Commons Attribution 4.0 International license. Further distribution of this work must maintain attribution to the author(s) and the published article's title, journal citation, and DOI.
}

define another set of multipole expansions:

$$
\boldsymbol{\rho}, \quad \boldsymbol{\rho} \cdot \mathbf{x}, \quad \sum_{e, f=1}^{d} \epsilon^{a b \cdots e f} \rho^{e} x^{f}, \ldots,
$$

where the third one is angular moment. $\epsilon^{a b \cdots}$ is the Levi-Civita symbol. If $d=2,3$, it can be rewritten in a compact form: $\boldsymbol{\rho} \times \mathbf{x}$. In $d=2, \boldsymbol{\rho} \times \mathbf{x}=\rho^{1} x^{2}-\rho^{2} x^{1}$.

Indeed, recently we have been witnesses to ongoing research progress on higher moment conservation and the associated higher rank version of global symmetry [2-8], especially in the field of fracton physics [2-66]. Some typical examples of research include subdiffusive transport at late times, nonergodicity, Hilbert space fragmentation, and spontaneous symmetry breaking $[29,31,38,65-70]$. In a simple scalar theory, the associated higher rank symmetry transformations are parametrized by $\theta(\mathbf{x})$ that is a polynomial function of $\mathbf{x}$ [7]. Inspired by the conventional correspondence between global symmetry and gauge symmetry, upon "gauging" higher rank symmetry, higher rank gauge fields can be obtained [4]. Here, the gauge fields are usually higher rank symmetric tensor fields, which leads to generalized Maxwell equations [5] and exotic theory of spin systems in Yb-based breathing pyrochlores [71].

As a nontrivial consequence of higher moment conservation, the mobility of particles is inevitably restricted, either partially or completely. For example, it is quite intuitive that dipole conservation strictly forbids a single particle motion along all spatial directions. Such particles are called "fractons" or 0-dimensional particles [2,3]. Similarly, one can define lineons (1-dimensional particle) that are movable within a stack of parallel straight lines and planons 
(2-dimensional particle) that are movable within a stack of parallel planes. Regarding these strange particles as bosons, we can consider their Bose-Einstein condensation, such that the spontaneous breaking of higher rank symmetry occurs. As a result, a class of exotic quantum phases of matter dubbed fractonic superfluids [65,66] is formed. In Ref. [66], a convenient notation $d \mathrm{SF}^{i}$ was introduced to denote $d$-dimensional fractonic superfluids with $i$-dimensional particle condensation, e.g., $d \mathrm{SF}^{0}$ with condensed fractons and $d \mathrm{SF}^{1}$ with condensed lineons. The conventional superfluid phase corresponds to $d \mathrm{SF}^{d}$ where bosons can freely move.

Higher-rank symmetric microscopic models often look quite unrealistic, highly anisotropic, and fine-tuned [4]. For example, Hamiltonian does not has the usual kinetic energy term [65]. And interaction is delicately designed [66]. However, as we have known, in many condensed matter systems, symmetry has been found to be significantly enhanced at low energies. For example, Lorentz symmetry emerges in graphene which is microscopically built by nonrelativistic electrons. Thus, one may wonder whether it is possible that the long-wavelength low-energy limit will conserve higher moments and respect higher rank symmetry as an emergent phenomenon.

For this purpose, we may apply the traditional theoretical approach: renormalization group (RG). If there exists a phase region such that all models in the region can flow to symmetric points, we can regard higher rank symmetry as an emergent symmetry. Theoretically, one advantage of such an emergent higher rank symmetry is its robustness against symmetry-breaking perturbation. Practically, we expect that such a scenario holds promise for more flexible realization of exotic higher rank symmetry and higher moment conservation in both theoretical and experimental studies.

In this paper, we identify such a wide phase region that supports emergent higher rank symmetry and conservation of angular moments, i.e., $\int d^{2} x \rho \times \mathbf{x}=\int d^{2} x\left(\rho^{1} x^{2}-\rho^{2} x^{1}\right)$ for a two-component boson field in two dimensions. We start with a two-dimensional many-boson system in the normal state (i.e., without lineon condensation) of fractonic superfluids (denoted as $2 \mathrm{SF}^{1}$ ). The Hamiltonian is a symmetric point in the parameter space where the $a$ th $(a=1,2)$ component bosons only have kinetic terms along $a$ th axis (dubbed "diagonal" kinetic terms). There also exists a weak intercomponent scattering term allowed by higher rank symmetry. We shall perform a RG analysis in the vicinity of the symmetric point by adding symmetry-disallowed kinetic terms (dubbed "off-diagonal" kinetic terms) as a perturbation. The one-loop calculation of the $\beta$ function shows that there exists a finite phase region (Fig. 4) where off-diagonal kinetic terms tend to be irrelevant under RG iteration. In other words, the highenergy model, which is not symmetric but more realistic and less fine-tuned, has a tendency to flow to the symmetric point. As a result, higher rank symmetry as well as conservation of angular moments emerges.

The remainder of this paper is organized as follows. In Sec. II, we introduce the $d$-component bosonic systems and their higher rank symmetry. In Sec. III, we discuss the scaling and Feynamn rules of the $d$-component bosonic systems. Further, we figure out the $\beta$ functions of parameters in the systems with renormalization group (RG) analysis and depict the global phase diagram. In Sec. IV, we summarize and provide our prediction on conditions of possible realization of systems with higher rank symmetry.

\section{MODEL AND SYMMETRY}

The symmetric point Hamiltonian for $d$-component bosonic systems in real space [66] is given by $\mathcal{H}=\mathcal{H}_{0}+\mathcal{H}_{1}$ with

$$
\begin{gathered}
\mathcal{H}_{0}=\sum_{a=1}^{d}\left[\frac{t_{a}}{2}\left(\partial_{a} \hat{\Phi}_{a}^{\dagger}\right)\left(\partial_{a} \hat{\Phi}_{a}\right)-\mu \hat{\Phi}_{a}^{\dagger} \hat{\Phi}_{a}\right] \\
\mathcal{H}_{1}=\frac{1}{2} \sum_{a \neq b} K_{a b}\left(\hat{\Phi}_{a}^{\dagger} \partial_{a} \hat{\Phi}_{b}^{\dagger}+\hat{\Phi}_{b}^{\dagger} \partial_{b} \hat{\Phi}_{a}^{\dagger}\right)\left(\hat{\Phi}_{a} \partial_{a} \hat{\Phi}_{b}+\hat{\Phi}_{b} \partial_{b} \hat{\Phi}_{a}\right) .
\end{gathered}
$$

Here $t_{a}=m_{a}^{-1}$ stands for the inverse of mass along the $a$ th direction. $\Phi_{a}^{\dagger}(\mathbf{x})$ and $\Phi_{a}(\mathbf{x})$ are respectively creation and annihilation operators, and satisfy the bosonic commutation relations. The interaction strength $K_{a b}$ is a symmetric matrix with vanishing diagonal elements, i.e., $K_{a a}=0, K_{a b}=K_{b a}$. Each term in $\mathcal{H}$ is invariant under both the conventional global symmetry transformations $\left(\hat{\Phi}_{a} \rightarrow e^{i \theta_{a}} \hat{\Phi}_{a}, \theta_{a} \in \mathbb{R}\right)$ and higher rank symmetry transformations:

$$
\left(\hat{\Phi}_{a}, \hat{\Phi}_{b}\right) \longrightarrow\left(\hat{\Phi}_{a} e^{i \lambda_{a b} x^{b}}, \hat{\Phi}_{b} e^{i \lambda_{b a} x^{a}}\right)
$$

for each pair $\left(\phi_{a}, \phi_{b}\right)$ with $\lambda_{a b}=-\lambda_{b a} \in \mathbb{R}$. According to Noether's theorem, the conventional global U(1) symmetry and the higher rank symmetry correspond to conserved total charge (particle number) $\mathcal{Q}^{a}$ and conserved total angular moments $\mathcal{Q}^{a b}\left(\mathcal{Q}^{a b}=-\mathcal{Q}^{b a}\right)[4,66]$ :

$$
\mathcal{Q}^{a}=\int d^{d} x \rho^{a}, \quad \mathcal{Q}^{a b}=\int d^{d} x\left(\rho^{a} x^{b}-\rho^{b} x^{a}\right) .
$$

Here $\rho^{a}=\hat{\Phi}_{a}^{\dagger} \hat{\Phi}_{a}$. Intuitively, the conserved quantities $\mathcal{Q}^{a b}$ enforce that a single $a$ th component boson can only move along the $a$ th direction. More explanation on the classical mechanical consequence of the conservation is available in Appendix A and Ref. [66].

In the coherent-state path-integral formulation with imaginary time, the Lagrangian density $\mathcal{L}$ can be written as $\mathcal{L}=\phi_{a}^{*} \partial_{\tau} \phi_{a}+\mathcal{H}$ with action $S=\int d \tau d^{d} x \mathcal{L}$. Here the bosonic fields $\phi_{a}=\phi_{a}(\mathbf{x}, \tau) \in \mathbb{C}$ are the eigenvales of coherent-state operators $\hat{\Phi}_{a}(\mathbf{x}, \tau)$. The Fourier transformation of the coherent state is $\phi_{a}(\tau, \mathbf{r})=$ $\frac{1}{\sqrt{\beta}} \sum_{n} \int \frac{d^{d} k}{(2 \pi)^{d}} e^{i \mathbf{k} \cdot \mathbf{r}-i \omega_{n} \tau} \phi_{a}\left(i \omega_{n}, \mathbf{k}\right)$ and its complex conjugate is $\quad \phi_{a}^{*}(\tau, \mathbf{r})=\frac{1}{\sqrt{\beta}} \sum_{n} \int \frac{d^{d} k}{(2 \pi)^{d}} e^{-i \mathbf{k} \cdot \mathbf{r}+i \omega_{n} \tau} \phi_{a}^{*}\left(i \omega_{n}, \mathbf{k}\right) \quad$ for $a=1,2, \ldots, d$. In the frequency-momentum space,

$$
\begin{aligned}
S= & \sum_{a ; k}\left(-i \omega_{n}+\xi_{a}\right) \phi_{a}^{*} \phi_{a}+\sum_{\substack{1,2,3,4 \\
a \neq b}} \frac{K_{a b}}{2 \beta}\left(k_{2}^{a}+k_{1}^{b}\right)\left(k_{3}^{a}+k_{4}^{b}\right) \\
& \times \phi_{1 a}^{*} \phi_{2 b}^{*} \phi_{3 b} \phi_{4 a} \delta(1+2-3-4) .
\end{aligned}
$$

In the first term on the right-hand side, the simplified notation $\phi_{a}$ stands for $\phi_{a}\left(i \omega_{n}, \mathbf{k}\right)$, which is the frequency-momentum image of the field $\phi_{a}(\mathbf{x}, \tau) . \omega_{n}$ is a bosonic Matsubara frequency and $\mathbf{k}$ is a momentum vector: $\mathbf{k}=\left(k^{1}, k^{2}, \ldots, k^{d}\right)$. We use $k^{a}$ to denote the $a$ th spatial component of momentum vector k. $\sum_{k}$ stands for $\sum_{\omega_{n}} \int \frac{d^{d} k}{(2 \pi)^{d}}$. In the second term, since 
there are four pairs of frequency and momentum, we introduce a new notation $\phi_{i a}$ to compactly represent $\phi_{a}\left(i \omega_{n_{i}}, \mathbf{k}_{i}\right)$ where the label $i=1,2, \ldots, 4$. $k_{i}^{a}$ stands for the $a$ th spatial component of momentum vector $\mathbf{k}_{i}$. The sum $\sum_{1,2,3,4}$ denotes $\sum_{i=1}^{4} \sum_{\omega_{n_{i}}} \int \frac{d^{d} k_{i}}{(2 \pi)^{d}}$. Other notations like $\sum_{1,2}, \sum_{2}, \ldots$ in the forthcoming text are defined in the similar way. Besides, the kinetic energy with momentum $\mathbf{k}$ is anisotropic: $\xi_{a}=\frac{1}{2} \sum_{a^{\prime}=1}^{2} t_{a a^{\prime}}\left(k^{a^{\prime}}\right)^{2}-\mu$. For momentum $\mathbf{k}_{i}$, the associated kinetic energy is $\xi_{i a}=\frac{1}{2} \sum_{b=1}^{d} t_{a b}\left(k_{i}^{b}\right)^{2}-\mu$. Last, we use $\delta(1+2-3-4)$ to represent $\delta_{\left(n_{1}+n_{2}\right),\left(n_{3}+n_{4}\right)}(2 \pi)^{d} \delta\left(\mathbf{k}_{\mathbf{1}}+\right.$ $\left.\mathbf{k}_{\mathbf{2}}-\mathbf{k}_{\mathbf{3}}-\mathbf{k}_{\mathbf{4}}\right) . \delta_{\left(n_{1}+n_{2}\right),\left(n_{3}+n_{4}\right)}$ is a dimensionless Kronecker symbol.

Before moving forward, we perturb the Lagrangian density by adding small "off-diagonal" kinetic terms that break higher rank symmetry. As such, kinetic terms of both directions are present, which can be written as $\sum_{a, b=1}^{d}\left[\frac{t_{a b}}{2}\left(\partial_{b} \phi_{a}^{*}\right)\left(\partial_{b} \phi_{a}\right)\right]=$ $\sum_{a=1}^{d}\left[\frac{t_{a a}}{2}\left(\partial_{a} \phi_{a}^{*}\right)\left(\partial_{a} \phi_{a}\right)\right]+\sum_{a \neq b}^{d}\left[\frac{t_{a b}}{2}\left(\partial_{b} \phi_{a}^{*}\right)\left(\partial_{b} \phi_{a}\right)\right]$. The kinetic parameter $t_{a}$ is rewritten as $t_{a a}$ for notational convenience. Those off-diagonal kinetic terms with nonzero $t_{a b}(a \neq b)$ manifestly break higher rank symmetry. Similarly, we can also understand these parameters as inverse of mass of field configuration $\phi_{a}$ along directions other than the $a$ th one: $t_{a b}=1 / m_{a b}$.

\section{RENORMALIZATION GROUP ANALYSIS}

\section{A. Scaling and Feynman rules}

We consider $d=2$. We set the restriction of field configuration $\phi_{a}$ as $\sum_{b=1}^{2} \frac{t_{a b}}{t_{a}}\left(k^{b}\right)^{2} \leqslant \Lambda^{2}$ since our total kinetic energy is given by $\sum_{b} \frac{t_{a b}}{2}\left(k^{b}\right)^{2}$ with surfaces of equal energy, $\sum_{b} \frac{t_{a b}}{t_{a a}}\left(k^{b}\right)^{2}=\kappa^{2}$. Here $\kappa$ is an arbitrary constant with the momentum dimension. The high-energy part corresponds to $\sqrt{\sum_{b=1}^{2} \frac{t_{a b}}{t_{a}}\left(k^{b}\right)^{2}} \in[\Lambda / s, \Lambda]$, where the scaling parameter $s>1$ and sends $\mathbf{k}$ to $\mathbf{k} / s$. We also define $s=e^{l}$ with $l>0$. We consider the free part of the action $\sum_{n} \int \frac{d^{2} k}{(2 \pi)^{2}} \phi_{a}^{*}\left(-i \omega_{n}+\right.$ $\left.\sum_{b=1}^{2} \frac{t_{a b}\left(k^{b}\right)^{2}}{2}\right) \phi_{a}$. It is noticed that we do not take into account the part related to chemical potential since it must be relevant if we choose the kinetic part to be marginal. Suppose the scaling dimension of $\phi$ is $\Delta_{\phi}$, and we can assume the change of temperature and frequencies are described as $T \rightarrow$ $s^{-z} T$ and $\omega_{n} \rightarrow s^{-z} \omega_{n}, n \in \mathbb{Z}$ respectively. So the free part is scaled to $\sum_{n} \int \frac{d^{2} k}{(2 \pi)^{2}} \phi_{a}^{*}\left(-i \omega_{n} s^{-z}+\sum_{b=1}^{2} \frac{t_{a b}\left(k^{b}\right)^{2}}{2} s^{-2}\right) \phi_{a} s^{2 \Delta_{\phi}-2}$. To fix the momentum dependence, we need $\Delta_{\phi}=\frac{d}{2}+1=$ 2 and $z=2$. In the $d=2$ case considered here, the interaction matrix is simply determined by a single parameter, i.e., $K_{12}=K_{21}:=\mathcal{K}$. To further simplify the calculation for $d=2$, we assume $t_{1}:=t_{11}=t_{2}:=t_{22}:=\mathcal{T}_{0}, t_{12}=t_{21}:=\mathcal{T}_{1}$. There are two small parameters compared to $\mathcal{T}_{0}$. The first one is the symmetry-disallowed off-diagonal kinetic parameter $\mathcal{T}_{1}$, which is marginal. It can also been seen as a perturbation relative to the interaction parameter $\mathcal{K} \Lambda^{2}$. The second one is the irrelevant interaction parameter $\mathcal{K} \Lambda^{2}$ considered as an infinitesimal quantity compared with the diagonal kinetic parameter $\mathcal{T}_{0}$. Hence, the following calculation will proceed
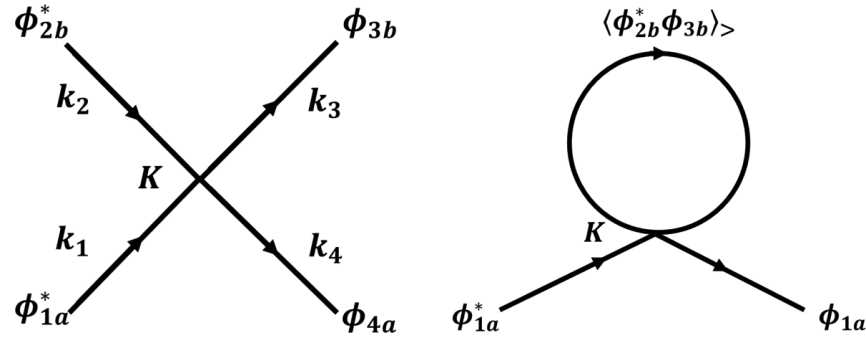

FIG. 1. The left diagram shows the bare interaction vertex of $d$-component bosons. Here the solid lines are Feynman propagators and the vertex is the coefficient: $\frac{1}{2 \beta} K_{a b}\left(k_{2}^{a}+k_{1}^{b}\right)\left(k_{3}^{a}+k_{4}^{b}\right) \delta(1+2-$ $3-4)$. The right one represents the Feynman loop diagram of the correction for the parameter $\mathcal{T}_{1}$.

in the perturbative regime: $\mathcal{T}_{0} \gg \mathcal{K} \Lambda^{2} \gg \mathcal{T}_{1}$, where $\Lambda$ is the momentum cutoff.

Next, we write Feynman rules. The bare Feynman propagators are given by $\left\langle\phi_{i a}^{*} \phi_{j b}\right\rangle=\left(i \omega_{n_{i}}-\xi_{i a}\right)^{-1} \delta_{a b} \delta_{i j}$. Here $\xi_{i a}=$ $\frac{\sum_{a^{\prime}=1}^{d} t_{a a^{\prime}}\left(k_{i}^{a^{\prime}}\right)^{2}}{2}-\mu$ stands for the kinetic energy of $\phi_{i a}$ [see definition below Eq. (7)]. In addition, the interaction vertex is drawn in Fig. 1. This diagram represents the interaction term, i.e., the second term in Eq. (7), where solid lines represent the bosonic fields and vertex stands for the interaction coefficient. In the RG procedure to be performed, we apply the standard cumulant expansion and relate the mean of the exponential to the exponential of the means [72,73]: $\left\langle e^{\left.-S_{\text {int }}\right\rangle_{>}}=e^{-\left\langle S_{\text {int }}\right\rangle_{>}+\frac{1}{2}\left(\left\langle S_{\text {int }}^{2}\right\rangle_{>}-\left\langle S_{\text {int }}\right\rangle_{>}^{2}\right)+\cdots}\right.$. Here $\phi_{>}$and $\phi_{<}$respectively correspond to the fast modes and slow modes of bosonic fields. In this case, the notation \langle\rangle$_{>}$is to take the average over the fast modes. Therefore, after calculating the average on fast modes, we have the form of the effective action: $S_{\text {eff }}\left[\phi_{<}\right]=S_{0}\left[\phi_{<}\right]+S_{\text {int }}^{\prime}\left[\phi_{<}\right]=S_{0}\left[\phi_{<}\right]+\left\langle S_{\text {int }}\right\rangle_{>}\left[\phi_{<}\right]-$ $\frac{1}{2}\left(\left\langle S_{\text {int }}^{2}\right\rangle_{>}-\left\langle S_{\text {int }}\right\rangle_{>}^{2}\right)\left[\phi_{<}\right]$. The form of $\left\langle S_{\text {int }}\right\rangle_{>}$contributes to the $\beta$ functions of kinetic parameters, also named first-order correction. Further, $\frac{1}{2}\left(\left\langle S_{\text {int }}^{2}\right\rangle_{>}-\left\langle S_{\text {int }}\right\rangle_{>}^{2}\right)\left[\phi_{<}\right]$leads to the $\beta$ functions of the elements in the $K$ matrix.

\section{B. One-loop correction to $\mathcal{T}_{1}$}

The interaction term reads

$$
\begin{aligned}
S_{\text {int }}= & \frac{1}{2 \beta} \sum_{1,2,3,4} \sum_{a \neq b} K_{a b}\left(k_{2}^{a}+k_{1}^{b}\right)\left(k_{3}^{a}+k_{4}^{b}\right) \phi_{1 a}^{*} \phi_{2 b}^{*} \phi_{3 b} \phi_{4 a} \\
& \times \delta(1+2-3-4) .
\end{aligned}
$$

Below we determine the scaling of the $K$ matrix. To be specific, we define the new momentum and frequency as $\mathbf{k}^{\prime}=s \mathbf{k}, \omega_{n}^{\prime}=s^{z} \omega_{n}$, or $T^{\prime}=s^{z} T$ with $z=2$. We define the rescaled field $\phi_{a}^{\prime}\left(i \omega_{n}^{\prime}, \mathbf{k}^{\prime}\right)=s^{-\Delta_{\phi}} \phi_{a}\left(i \omega_{n}^{\prime} s^{-z}, \frac{\mathbf{k}^{\prime}}{s}\right)$ with $\Delta_{\phi}=\frac{d}{2}+1$. Then the scaling of parameters $K_{a b}$ can be determined. With the form of interacting action (8), 
we obtain

$$
\begin{aligned}
S_{\mathrm{int}}^{\prime}= & \frac{1}{2 \beta^{\prime}} s^{-z} \sum_{i=1}^{4} \sum_{\omega_{n_{i}}^{\prime}} \int \frac{d^{d} k_{i}^{\prime}}{(2 \pi)^{d}} s^{-4 d} K_{a b}\left[\left(k_{2}^{\prime}\right)^{a}+\left(k_{1}^{\prime}\right)^{b}\right]\left[\left(k_{3}^{\prime}\right)^{a}+\left(k_{4}^{\prime}\right)^{b}\right] s^{-2} \\
& \times \phi_{a}^{\prime *}\left(i \omega_{n_{1}}^{\prime}, \mathbf{k}_{1}^{\prime}\right) \phi_{b}^{\prime *}\left(i \omega_{n_{2}}^{\prime}, \mathbf{k}_{2}^{\prime}\right) \phi_{b}^{\prime}\left(i \omega_{n_{3}}^{\prime}, \mathbf{k}_{3}^{\prime}\right) \phi_{a}^{\prime}\left(i \omega_{n_{4}}^{\prime}, \mathbf{k}_{4}^{\prime}\right) s^{4 \Delta_{\phi}} \delta_{\left(n_{1}^{\prime}+n_{2}^{\prime}\right),\left(n_{3}^{\prime}+n_{4}^{\prime}\right)}(2 \pi)^{d} \delta\left(\mathbf{k}_{\mathbf{1}}^{\prime}+\mathbf{k}_{2}^{\prime}-\mathbf{k}_{\mathbf{3}}^{\prime}-\mathbf{k}_{\mathbf{4}}^{\prime}\right) s^{d} \\
= & \frac{1}{2 \beta^{\prime}} s^{2-z-d} \sum_{i=1}^{4} \sum_{\omega_{n_{i}}^{\prime}} \int \frac{d^{d} k_{i}^{\prime}}{(2 \pi)^{d}} \sum_{a \neq b} K_{a b}\left[\left(k_{2}^{\prime}\right)^{a}+\left(k_{1}^{\prime}\right)^{b}\right]\left[\left(k_{3}^{\prime}\right)^{a}+\left(k_{4}^{\prime}\right)^{b}\right] \\
& \times \phi_{a}^{\prime *}\left(i \omega_{n_{1}}^{\prime}, \mathbf{k}_{1}^{\prime}\right) \phi_{b}^{\prime *}\left(i \omega_{n_{2}}^{\prime}, \mathbf{k}_{2}^{\prime}\right) \phi_{b}^{\prime}\left(i \omega_{n_{3}}^{\prime}, \mathbf{k}_{3}^{\prime}\right) \phi_{a}^{\prime}\left(i \omega_{n_{4}}^{\prime}, \mathbf{k}_{4}^{\prime}\right) \delta_{\left(n_{1}^{\prime}+n_{2}^{\prime}\right),\left(n_{3}^{\prime}+n_{4}^{\prime}\right)}(2 \pi)^{d} \delta\left(\mathbf{k}_{\mathbf{1}}^{\prime}+\mathbf{k}_{\mathbf{2}}^{\prime}-\mathbf{k}_{\mathbf{3}}^{\prime}-\mathbf{k}_{\mathbf{4}}^{\prime}\right)
\end{aligned}
$$

Here the dimensionful $\delta$ function of momenta is also scaled $\left[\delta\left(\mathbf{k}^{\prime}\right)=s^{-d} \delta(\mathbf{k})\right]$ but the Kronecker symbol of Matsubara frequencies is dimensionless and invariant upon scaling. Therefore, the scaling of $K_{a b}$ is given by $K_{a b}^{\prime}=K_{a b} s^{-d}$. It illustrates that the $K$ matrix at tree level is irrelevant in perturbative RG.

Then, let us consider the first-order correction to $\mathcal{T}_{1}$, $\left\langle S_{\text {int }}\right\rangle_{>}$, contributing to the correction of kinetic parameters:

$$
\begin{aligned}
\left\langle S_{\text {int }}\right\rangle_{>}= & \left\langle\frac{1}{2 \beta} \sum_{1,2,3,4} \sum_{a \neq b} K_{a b}\left(k_{2}^{a}+k_{1}^{b}\right)\left(k_{3}^{a}+k_{4}^{b}\right)\right. \\
& \left.\times \phi_{1 a}^{*} \phi_{2 b}^{*} \phi_{3 b} \phi_{4 a} \delta(1+2-3-4)\right\rangle_{>} .
\end{aligned}
$$

To proceed further, we should split each momentum integration into a slow part $(<)$ and a fast part $(>)$. In this way, the integration of four momenta $\left(\mathbf{k}_{1}, \mathbf{k}_{2}, \mathbf{k}_{3}, \mathbf{k}_{4}\right)$ is split into $2^{4}=16$ combinations. According to Wick's theorem, for formulas of bare propagators, we conclude that either $\phi_{1 a}^{*}$ and $\phi_{4 a}$ must be paired or $\phi_{2 b}^{*}$ and $\phi_{3 b}$ must be paired. Other contractions vanish due to $K_{a b}=0$ when $a=b$. Therefore, of 16 combinations, only the following two are nonvanishing:

Case 1: The momenta carried by $\phi_{1 a}^{*}$ and $\phi_{4 a}$, i.e., $\mathbf{k}_{1}$ and $\mathbf{k}_{4}$, are fast momenta. ${ }^{1} \mathbf{k}_{1}=\mathbf{k}_{4}$ and $\omega_{n_{1}}=\omega_{n_{4}}$ are required by the formula of the bare propagator;

Case II: The momenta carried by $\phi_{2 b}^{*}$ and $\phi_{3 b}$, i.e., $\mathbf{k}_{2}$ and $\mathbf{k}_{3}$, are fast momenta. $\mathbf{k}_{2}=\mathbf{k}_{3}$ and $\omega_{n_{2}}=\omega_{n_{3}}$ are required by the formula of the bare propagator.

By further considering $\delta(1+2-3-4)$, we have

Case I: The momenta carried by $\phi_{1 a}^{*}$ and $\phi_{4 a}$, i.e., $\mathbf{k}_{1}$ and $\mathbf{k}_{4}$, are fast momenta. $\mathbf{k}_{1}=\mathbf{k}_{4}$ and $\omega_{n_{1}}=\omega_{n_{4}} ; \mathbf{k}_{2}=\mathbf{k}_{3}$ and $\omega_{n_{2}}=\omega_{n_{3}}$.

Case II: The momenta carried by $\phi_{2 b}^{*}$ and $\phi_{3 b}$, i.e., $\mathbf{k}_{2}$ and $\mathbf{k}_{3}$, are fast momenta. $\mathbf{k}_{2}=\mathbf{k}_{3}$ and $\omega_{n_{2}}=\omega_{n_{3}} ; \mathbf{k}_{1}=\mathbf{k}_{4}$ and $\omega_{n_{1}}=\omega_{n_{4}}$.

These two contractions correspond to the Feynman diagram in Fig. 1. We first focus on case I. When 1,4 are fast modes, the momentum conservation and Feynman rules will tell us $\mathbf{k}_{2}=\mathbf{k}_{3}, \mathbf{k}_{1}=\mathbf{k}_{4} ; \omega_{n_{2}}=\omega_{n_{3}}, \omega_{n_{1}}=\omega_{n_{4}}$. Since we

\footnotetext{
${ }^{1}$ By fast (slow) momentum, we mean that the kinetic energy corresponding to the momentum is large (small).
}

focus on the two-dimensional case $(d=2)$, we set all offdiagonal $K$-matrix elements as $K_{12}=K_{21}:=\mathcal{K}$. To further simplify the calculation, we assume $t_{1}:=t_{11}=t_{2}:=t_{22}:=$ $\mathcal{T}_{0}$ and $t_{12}=t_{21}:=\mathcal{T}_{1}$. In this way, $\left\langle S_{\mathrm{int}}\right\rangle_{>}^{I}$ is given by

$$
\begin{aligned}
\left\langle S_{\text {int }}\right\rangle_{>}^{I}= & \frac{1}{2 \beta} \sum_{a \neq b} \sum_{1,2} \mathcal{K}\left(k_{2}^{a}+k_{1}^{b}\right)^{2}\left\langle\phi_{1 a}^{*} \phi_{1 a}\right\rangle_{>} \phi_{2 b}^{*} \phi_{2 b} \\
= & \frac{1}{2 \beta} \sum_{a \neq b} \sum_{1,2} \mathcal{K}\left(k_{2}^{a}+k_{1}^{b}\right)^{2} \frac{1}{i \omega_{n_{1}}-\xi_{1 a}} \phi_{2 b}^{*} \phi_{2 b} \\
= & -\frac{1}{2} \sum_{a \neq b} \sum_{2} \int_{>} \frac{d^{2} k_{1}}{(2 \pi)^{2}} \mathcal{K}\left(k_{2}^{a}+k_{1}^{b}\right)^{2} f_{B}\left(\xi_{1 a}\right) \phi_{2 b}^{*} \phi_{2 b} \\
= & -\frac{1}{2} \sum_{a \neq b} \sum_{2} \int_{>} \frac{d^{2} k_{1}}{(2 \pi)^{2}} \mathcal{K}\left[\left(k_{2}^{a}\right)^{2}+2 k_{2}^{a} k_{1}^{b}+\left(k_{1}^{b}\right)^{2}\right] \\
& \times f_{B}\left(\xi_{1 a}\right) \phi_{2 b}^{*} \phi_{2 b},
\end{aligned}
$$

where $\mathbf{k}_{1}$ and $\mathbf{k}_{2}$ correspond to fast and slow momenta respectively. The bosonic Matsubara summation is applied: $\frac{1}{\beta} \sum_{\omega_{n}} \frac{1}{i \omega_{n}-\xi_{1 a}}=-f_{B}\left(\xi_{1 a}\right)=-\frac{1}{e^{\beta \xi_{1 a}-1}}$. And the propagator $\left\langle\phi_{1 a}^{*} \phi_{1 a}\right\rangle_{>}$is given by $\left\langle\phi_{1 a}^{*} \phi_{1 a}\right\rangle_{>}=\frac{1}{i \omega_{n_{1}}-\xi_{1 a}}$ with $\xi_{1 a}(a=$ $1, b=2$ or $a=2, b=1)$ :

$$
\xi_{1 a}:=\frac{\mathcal{T}_{0}\left(k_{1}^{a}\right)^{2}+\mathcal{T}_{1}\left(k_{1}^{b}\right)^{2}}{2}-\mu .
$$

In the same way, we can give the form of case II:

$$
\begin{aligned}
\left\langle S_{\text {int }}\right\rangle_{>}^{I I}= & \frac{1}{2 \beta} \sum_{a \neq b} \sum_{1,2} \mathcal{K}\left(k_{2}^{a}+k_{1}^{b}\right)^{2}\left\langle\phi_{2 b}^{*} \phi_{2 b}\right\rangle_{>} \phi_{1 a}^{*} \phi_{1 a} \\
= & \frac{1}{2 \beta} \sum_{a \neq b} \sum_{1,2} \mathcal{K}\left(k_{2}^{a}+k_{1}^{b}\right)^{2} \frac{1}{i \omega_{n_{2}}-\xi_{2 b}} \phi_{1 a}^{*} \phi_{1 a} \\
= & -\frac{1}{2} \sum_{a \neq b} \sum_{1} \int_{>} \frac{d^{2} k_{2}}{(2 \pi)^{2}} \mathcal{K}\left(k_{2}^{a}+k_{1}^{b}\right)^{2} f_{B}\left(\xi_{2 b}\right) \phi_{1 a}^{*} \phi_{1 a} \\
= & -\frac{1}{2} \sum_{a \neq b} \sum_{1} \int_{>} \frac{d^{2} k_{2}}{(2 \pi)^{2}} \mathcal{K}\left[\left(k_{2}^{a}\right)^{2}+2 k_{2}^{a} k_{1}^{b}+\left(k_{1}^{b}\right)^{2}\right] \\
& \times f_{B}\left(\xi_{2 b}\right) \phi_{1 a}^{*} \phi_{1 a} .
\end{aligned}
$$

By observing these two actions, we find they are completely equivalent to each other by exchanging the indices $(a \leftrightarrow$ $b, 1 \leftrightarrow 2$ ). Then we focus on case I and multiply it by 2 , 
namely,

$$
\begin{aligned}
\left\langle S_{\mathrm{int}}\right\rangle_{>}= & \left\langle S_{\mathrm{int}}\right\rangle_{>}^{I}+\left\langle S_{\mathrm{int}}\right\rangle_{>}^{I I}=2\left\langle S_{\mathrm{int}}\right\rangle_{>}^{I} \\
= & -\sum_{a \neq b} \sum_{2} \int_{>} \frac{d^{2} k_{1}}{(2 \pi)^{2}} \mathcal{K}\left[\left(k_{2}^{a}\right)^{2}+2 k_{2}^{a} k_{1}^{b}+\left(k_{1}^{b}\right)^{2}\right] \\
& \times f_{B}\left(\xi_{1 a}\right) \phi_{2 b}^{*} \phi_{2 b}=\left\langle S_{\mathrm{int}}\right\rangle_{>}^{(1)}+\left\langle S_{\mathrm{int}}\right\rangle_{>}^{(2)}+\left\langle S_{\mathrm{int}}\right\rangle_{>}^{(3)},
\end{aligned}
$$

where we arrange all terms into three parts:

$$
\begin{aligned}
\left\langle S_{\text {int }}\right\rangle_{>}^{(1)} & :=-\sum_{a \neq b} \sum_{2} \mathcal{K}\left(k_{2}^{a}\right)^{2} \int_{>} \frac{d^{2} k_{1}}{(2 \pi)^{2}} f_{B}\left(\xi_{1 a}\right) \phi_{2 b}^{*} \phi_{2 b}, \\
\left\langle S_{\text {int }}\right\rangle_{>}^{(2)} & :=-2 \sum_{a \neq b} \sum_{2} \mathcal{K} k_{2}^{a} \int_{>} \frac{d^{2} k_{1}}{(2 \pi)^{2}} k_{1}^{b} f_{B}\left(\xi_{1 a}\right) \phi_{2 b}^{*} \phi_{2 b}, \\
\left\langle S_{\text {int }}\right\rangle_{>}^{(3)} & :=-\sum_{a \neq b} \sum_{2} \mathcal{K} \int_{>} \frac{d^{2} k_{1}}{(2 \pi)^{2}} f_{B}\left(\xi_{1 a}\right)\left(k_{1}^{b}\right)^{2} \phi_{2 b}^{*} \phi_{2 b} .
\end{aligned}
$$

The fast momentum $\mathbf{k}_{1}=\left(k_{1}^{1}, k_{1}^{2}\right)$ takes values in the elliptic shell $(\Lambda$ is the momentum cutoff and the RG flow parameter $s=e^{l}$ with $s \rightarrow 1$ and $l \rightarrow 0$ ):

$$
\left(\frac{\Lambda}{s}\right)^{2}<\left(k_{1}^{a}\right)^{2}+\frac{\mathcal{T}_{1}}{\mathcal{T}_{0}}\left(k_{1}^{b}\right)^{2}<\Lambda^{2},
$$

which defines the domain of integral $\int_{>}$. Here we should focus on the integrated domain of fast momenta. It is only determined by the fields carrying those fast momenta. To be specific, we consider the fast momentum $\mathbf{k}_{\mathbf{i}}$ carried by the fast mode $\phi_{i a}$ or $\phi_{i a}^{*}$ with the label $i=1,2, \ldots, 4$ defined above. Its integrated domain is given by $(\Lambda / s)^{2}<\left(k_{i}^{a}\right)^{2}+\frac{\mathcal{T}_{1}}{\mathcal{T}_{0}}\left(k_{i}^{b}\right)^{2}<$ $\Lambda^{2}$ for $b \neq a$. This conclusion will be used in the following text. To compute $\left\langle S_{\text {int }}\right\rangle_{>}^{(1)}$ in Eq. (13), we may introduce a new momentum $\tilde{\mathbf{k}}: \tilde{k}^{a}=k_{1}^{a}, \tilde{k}^{b}=\sqrt{\frac{\mathcal{T}_{1}}{\mathcal{T}_{0}}} k_{1}^{b}$. Then, Eq. (16) is changed to $\left(\frac{\Lambda}{s}\right)^{2}<\left(\tilde{k}^{a}\right)^{2}+\left(\tilde{k}^{b}\right)^{2}<\Lambda^{2}$, i.e., $\left(\frac{\Lambda}{s}\right)^{2}<\tilde{\mathbf{k}}^{2}<$ $\Lambda^{2}$. And Eq. (12) is changed to

$$
\xi_{1 a}=\frac{\mathcal{T}_{0}\left[\left(\tilde{k}^{a}\right)^{2}+\left(\tilde{k}^{b}\right)^{2}\right]}{2}-\mu=\frac{\mathcal{T}_{0} \tilde{\mathbf{k}}^{2}}{2}-\mu .
$$

Using the new momentum variables, we can work out the integral $\int_{>}$in $\left\langle S_{\text {int }}\right\rangle_{>}^{(1)}$ in Eq. (13) (s-1 is small enough):

$$
\begin{aligned}
& \int_{>} \frac{d^{2} k_{1}}{(2 \pi)^{2}} f_{B}\left(\xi_{1 a}\right) \\
& \quad=\sqrt{\frac{\mathcal{T}_{0}}{\mathcal{T}_{1}}} \int_{>} \frac{d^{2} \tilde{k}}{(2 \pi)^{2}} f_{B}\left(\xi_{1 a}\right) \\
& \quad=\sqrt{\frac{\mathcal{T}_{0}}{\mathcal{T}_{1}}} \frac{1}{(2 \pi)^{2}} \int_{\Lambda / s}^{\Lambda} 2 \pi|\tilde{\mathbf{k}}| d|\tilde{\mathbf{k}}| \frac{1}{e^{\beta \xi 1 a}-1} \\
& \quad=\sqrt{\frac{\mathcal{T}_{0}}{\mathcal{T}_{1}}} \frac{1}{(2 \pi)^{2}} \int_{(\Lambda / s)^{2}}^{\Lambda^{2}} \frac{2 \pi}{2} d\left(|\tilde{\mathbf{k}}|^{2}\right) \frac{1}{e^{\beta \xi_{1 a}}-1} \\
& \approx \sqrt{\frac{\mathcal{T}_{0}}{\mathcal{T}_{1}}} \frac{1}{(2 \pi)^{2}} \frac{2 \pi}{2} \frac{1}{e^{\beta \xi_{0 \Lambda}}-1}\left[\Lambda^{2}-\left(\frac{\Lambda}{s}\right)^{2}\right] \\
& \approx \sqrt{\frac{\mathcal{T}_{0}}{\mathcal{T}_{1}}} \frac{1}{(2 \pi)^{2}} \frac{2 \pi}{2} \frac{1}{e^{\beta \xi_{0 \Lambda}}-1} 2 \Lambda^{2} l=\sqrt{\frac{\mathcal{T}_{0}}{\mathcal{T}_{1}}} \frac{1}{2 \pi} \frac{1}{e^{\beta \xi_{0 \Lambda}}-1} \Lambda^{2} l,
\end{aligned}
$$

where we have considered infinitesimal $s-1$ and the definition of $s=e^{l}$. And, $\xi_{0 \Lambda}:=\frac{\mathcal{T}_{0}}{2} \Lambda^{2}-\mu$. As a result,

$$
\left\langle S_{\mathrm{int}}\right\rangle_{>}^{(1)}=-S_{2} f_{B}\left(\xi_{0 \Lambda}\right) \frac{\Lambda^{2}}{(2 \pi)^{2}} \sum_{a \neq b} \sum_{2} \mathcal{K}\left(k_{2}^{a}\right)^{2} \phi_{2 b}^{*} \phi_{2 b} l,
$$

where $S_{2}:=2 \pi \sqrt{\frac{\mathcal{T}_{0}}{\mathcal{T}_{1}}}$ and $f_{B}\left(\xi_{o \Lambda}\right):=\frac{1}{e^{\beta_{0 \Lambda}-1}}$. Below, we will use $d l$ to replace $l$ since it is infinitesimal.

The second part, $\left\langle S_{\text {int }}\right\rangle_{>}^{(2)}$ in Eq. (14), vanishes since it is an odd function of the integrated momentum $\mathbf{k}_{1}$. The third term in Eq. (15) is directly connected to correction of the chemical potential. We just briefly give our results here because it is not our focus:

$$
\left\langle S_{\text {int }}\right\rangle_{>}^{(3)}=-\frac{1}{2} \sum_{a \neq b} \sum_{2} \Lambda^{4} \frac{S_{2}}{(2 \pi)^{2}} f_{B}\left(\xi_{a \Lambda}\right) K \phi_{2 b}^{*} \phi_{2 b}\left(1-\frac{1}{s}\right) .
$$

Therefore, the only term that can renormalize $\mathcal{T}_{1}$ is $\left\langle S_{\text {int }}\right\rangle_{>}^{(1)}$ in Eq. (18). The bare off-diagonal kinetic term is given by $\sum_{a \neq b} \sum_{k} \frac{\mathcal{T}_{1}}{2}\left(k_{2}^{a}\right)^{2} \phi_{2 b}^{*} \phi_{2 b}$ in the frequency-momentum space. Hence, we have the $\beta$ function of the parameter $\mathcal{T}_{1}$ by referencing the effective action $S_{\text {eff }}\left[\phi_{<}\right]=S_{0}\left[\phi_{<}\right]+\left\langle S_{\text {int }}\right\rangle_{>}\left[\phi_{<}\right]-$ $\frac{1}{2}\left(\left\langle S_{\text {int }}^{2}\right\rangle_{>}-\left\langle S_{\text {int }}\right\rangle_{>}^{2}\right)\left[\phi_{<}\right]:$

$$
\frac{d \mathcal{T}_{1}}{d l}=-S_{2} \mathcal{K} f_{B}\left(\xi_{a \Lambda}\right) \frac{\Lambda^{2}}{(2 \pi)^{2}}=-\sqrt{\frac{\mathcal{T}_{0}}{\mathcal{T}_{1}}} \mathcal{K} f_{B}\left(\xi_{a \Lambda}\right) \frac{\Lambda^{2}}{2 \pi} .
$$

This corresponds to Fig. 1. Besides, by referencing the term containing the chemical potential, we have the $\beta$ function of the chemical potential $\mu$ : $\frac{d \mu}{d l}=2 \mu-\frac{1}{2} \Lambda^{4} \frac{S_{2}}{(2 \pi)^{2}} f_{B}\left(\xi_{a \Lambda}\right) \mathcal{K}$, with the term $2 \mu$ originating from the contribution from the tree-level diagrams.

\section{Vertex correction to $\mathcal{K}$}

We then turn to the vertex correction with all the contractions contained in $\left\langle S_{\text {int }}^{2}\right\rangle_{>}$:

$$
\begin{aligned}
\left\langle S_{\mathrm{int}}^{2}\right\rangle_{>}= & \frac{1}{4 \beta^{2}} \sum_{a \neq b} \sum_{a^{\prime} \neq b^{\prime}} \sum_{1,2,3,4} \sum_{1^{\prime}, 2^{\prime}, 3^{\prime}, 4^{\prime}} \\
& \times K_{a b} K_{a^{\prime} b^{\prime}}\left\langle\phi_{1 a}^{*} \phi_{2 b}^{*} \phi_{3 b} \phi_{4 a} \phi_{1^{\prime} a^{\prime}}^{*} \phi_{2^{\prime} b^{\prime}}^{*} \phi_{3^{\prime} b^{\prime}} \phi_{4^{\prime} a^{\prime}}\right\rangle_{>} \\
& \times\left(k_{2}^{a}+k_{1}^{b}\right)\left(k_{3}^{a}+k_{4}^{b}\right)\left(k_{2^{\prime}}^{a^{\prime}}+k_{1^{\prime}}^{b^{\prime}}\right)\left(k_{3^{\prime}}^{a^{\prime}}+k_{4^{\prime}}^{b^{\prime}}\right) \\
& \times \delta(1+2-3-4) \delta\left(1^{\prime}+2^{\prime}-3^{\prime}-4^{\prime}\right) \\
= & \left\langle S_{\text {int }}^{2}\right\rangle_{>}^{(1)}+\left\langle S_{\mathrm{int}}^{2}\right\rangle_{>}^{(2)} .
\end{aligned}
$$

Since we only consider the loop-diagram contribution, only two kinds of contractions will not vanish under calculation. The first term $\left\langle S_{\text {int }}^{2}\right\rangle_{>}^{(1)}$ can be considered in four cases:

Case 1: The momenta $\mathbf{k}_{\mathbf{1}}, \mathbf{k}_{\mathbf{2}}, \mathbf{k}_{\mathbf{3}^{\prime}}$, and $\mathbf{k}_{\mathbf{4}^{\prime}}$ are fast momenta. $\mathbf{k}_{\mathbf{1}}=\mathbf{k}_{\mathbf{3}^{\prime}}$ and $\omega_{n_{1}}=\omega_{n_{3^{\prime}}} ; \mathbf{k}_{\mathbf{2}}=\mathbf{k}_{\mathbf{4}^{\prime}}$ and $\omega_{n_{2}}=\omega_{n_{4^{\prime}}}$.

Case 2: The momenta $\mathbf{k}_{\mathbf{1}}, \mathbf{k}_{\mathbf{2}}, \mathbf{k}_{\mathbf{3}^{\prime}}$, and $\mathbf{k}_{\mathbf{4}^{\prime}}$ are fast momenta. $\mathbf{k}_{\mathbf{1}}=\mathbf{k}_{\mathbf{4}^{\prime}}$ and $\omega_{n_{1}}=\omega_{n^{4^{\prime}}} ; \mathbf{k}_{\mathbf{2}}=\mathbf{k}_{\mathbf{3}^{\prime}}$ and $\omega_{n_{2}}=\omega_{n_{3^{\prime}}}$.

Case 3: The momenta $\mathbf{k}_{\mathbf{1}^{\prime}}, \mathbf{k}_{\mathbf{2}^{\prime}}, \mathbf{k}_{\mathbf{3}}$, and $\mathbf{k}_{\mathbf{4}}$ are fast momenta. $\mathbf{k}_{\mathbf{1}^{\prime}}=\mathbf{k}_{\mathbf{3}}$ and $\omega_{n_{1^{\prime}}}=\omega_{n_{3}} ; \mathbf{k}_{\mathbf{2}^{\prime}}=\mathbf{k}_{\mathbf{4}}$ and $\omega_{n_{2^{\prime}}}=\omega_{n_{4}}$.

Case 4: The momenta $\mathbf{k}_{\mathbf{1}^{\prime}}, \mathbf{k}_{\mathbf{2}^{\prime}}, \mathbf{k}_{\mathbf{3}}$, and $\mathbf{k}_{\mathbf{4}}$ are fast momenta. $\mathbf{k}_{\mathbf{1}^{\prime}}=\mathbf{k}_{\mathbf{4}}$ and $\omega_{n_{1^{\prime}}}=\omega_{n_{4}} ; \mathbf{k}_{\mathbf{2}^{\prime}}=\mathbf{k}_{\mathbf{3}}$ and $\omega_{n_{2^{\prime}}}=\omega_{n_{3}}$.

These four cases correspond to the Feynman diagram in Fig. 2. Similarly, it can also be proved that these 
four cases are equal to one another by exchanging the indices. We directly take the case 1 as an example and multiply it by 4 for simplicity. By further considering the delta functions in the vertex correction, the relationships between momenta and frequencies are given by $\mathbf{k}_{1}=\mathbf{k}_{3^{\prime}}, \mathbf{k}_{2}=\mathbf{k}_{4^{\prime}}, \mathbf{k}_{1}+\mathbf{k}_{2}=\mathbf{k}_{3}+\mathbf{k}_{4}=$ $\mathbf{k}_{1^{\prime}}+\mathbf{k}_{2^{\prime}}=\mathbf{k}_{3^{\prime}}+\mathbf{k}_{4^{\prime}} ; \omega_{n_{1}}=\omega_{n_{3^{\prime}}}, \omega_{n_{2}}=\omega_{n_{4^{\prime}}}, \omega_{n_{1}}+\omega_{n_{2}}=$ $\omega_{n_{3}}+\omega_{n_{4}}=\omega_{n_{1^{\prime}}}+\omega_{n_{2^{\prime}}}=\omega_{n_{3^{\prime}}}+\omega_{n_{4^{\prime}}}$. Hence, with the formula (20), we obtain that

$$
\begin{aligned}
\left\langle S_{\mathrm{int}}^{2}\right\rangle_{>}^{(1)}= & \frac{1}{\beta^{2}} \sum_{a \neq b} \sum_{1,2,3,4} \sum_{1^{\prime}, 2^{\prime}} \mathcal{K}^{2}\left(k_{2}^{a}+k_{1}^{b}\right)^{2}\left(k_{3}^{a}+k_{4}^{b}\right)\left(k_{2^{\prime}}^{a^{\prime}}+k_{1^{\prime}}^{b^{\prime}}\right) \frac{1}{i \omega_{n_{1}}-\xi_{1 a}} \frac{1}{i \omega_{n_{2}}-\xi_{2 b}} \phi_{1^{\prime} a}^{*} \phi_{2^{\prime} b}^{*} \phi_{3 b} \phi_{4 a} \\
& \times \delta\left(1^{\prime}+2^{\prime}-3-4\right) \delta\left(1^{\prime}+2^{\prime}-2-1\right) \\
= & \frac{1}{\beta^{2}} \sum_{a \neq b} \sum_{2,3,4} \sum_{1^{\prime}, 2^{\prime}} \mathcal{K}^{2}\left(k_{2}^{a}-k_{2}^{b}+k_{1^{\prime}}^{b}+k_{2^{\prime}}^{b}\right)^{2}\left(k_{3}^{a}+k_{4}^{b}\right)\left(k_{2^{\prime}}^{a^{\prime}}+k_{1^{\prime}}^{b^{\prime}}\right) \\
& \times \frac{1}{i \omega_{n_{1^{\prime}}}+i \omega_{n_{2^{\prime}}}-i \omega_{n_{2}}-\xi_{1^{\prime}+2^{\prime}-2, a}} \frac{1}{i \omega_{n_{2}}-\xi_{2 b}} \phi_{1^{\prime} a}^{*} \phi_{2^{\prime} b}^{*} \phi_{3 b} \phi_{4 a} \delta\left(1^{\prime}+2^{\prime}-3-4\right) \\
= & -\frac{1}{\beta^{2}} \sum_{a \neq b} \int \frac{d^{2} k_{2}}{(2 \pi)^{2}} \sum_{3,4} \sum_{1^{\prime}, 2^{\prime}} \mathcal{K}^{2}\left(k_{2}^{a}-k_{2}^{b}+k_{1^{\prime}}^{b}+k_{2^{\prime}}^{b}\right)^{2}\left(k_{3}^{a}+k_{4}^{b}\right)\left(k_{2^{\prime}}^{a^{\prime}}+k_{1^{\prime}}^{b^{\prime}}\right) \\
& \times \sum_{n_{2}} \frac{1}{i \omega_{n_{2}}-i \omega_{n_{1^{\prime}}}-i \omega_{n_{2^{\prime}}}+\xi_{1^{\prime}+2^{\prime}-2, a}} \frac{1}{i \omega_{n_{2}}-\xi_{2 b}} \phi_{1^{\prime} a}^{*} \phi_{2^{\prime} b}^{*} \phi_{3 b} \phi_{4 a} \delta\left(1^{\prime}+2^{\prime}-3-4\right),
\end{aligned}
$$

where we introduce the notation $\xi_{1^{\prime}+2^{\prime}-2, a}=$ $\frac{\sum_{a^{\prime}=1}^{2} t_{a a^{\prime}}\left(k_{1^{\prime}}^{a^{\prime}}+k_{2^{\prime}}^{a^{\prime}}-k_{2}^{a^{\prime}}\right)^{2}}{2}-\mu$. This expression contains a sum of Matsubara frequencies $n_{2}: \sum_{n_{2}} \frac{1}{i \omega_{n_{2}}-i \omega_{n_{1^{\prime}}}-i \omega_{n_{2^{\prime}}}+\xi_{1^{\prime}+2^{\prime}-2, a}} \frac{1}{i \omega_{n_{2}}-\xi_{2 b}}$. By applying the formula $\frac{1}{\beta} \sum_{n} \frac{1}{i \omega_{n}-\xi_{1}} \frac{1}{i \omega_{n}-\xi_{2}}=-\frac{f_{B}\left(\xi_{1}\right)-f_{B}\left(\xi_{2}\right)}{\xi_{1}-\xi_{2}}$ for Matsubara frequencies for bosons, $\omega_{n}=\frac{2 \pi n}{\beta}$, we can obtain the result with substitutions: $\xi_{1}=i \omega_{n_{1^{\prime}}}+i \omega_{n_{2^{\prime}}}-$ $\xi_{1^{\prime}+2^{\prime}-2, a}$ and $\xi_{2}=\xi_{2 b}$. The sum of $n_{2}$ can be rewritten as

$$
\frac{1}{\beta} \sum_{n_{2}} \frac{1}{i \omega_{n_{2}}-i \omega_{n_{1^{\prime}}}-i \omega_{n_{2^{\prime}}}+\xi_{1^{\prime}+2^{\prime}-2, a}} \frac{1}{i \omega_{n_{2}}-\xi_{2 b}}
$$

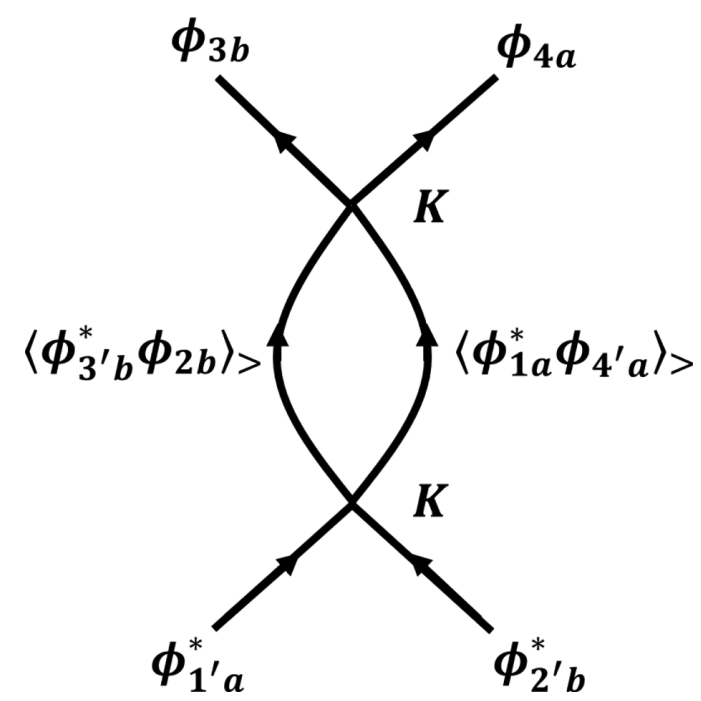

FIG. 2. The Feynman diagram of the first type of contraction in the vertex correction contributes to the correction of the parameter $\mathcal{K}$. It corresponds to cases $1-4$. For simplicity, we here show one of the related Feynman diagrams. By exchanging the symmetric indices, we can obtain the other three possible diagrams.

$$
\begin{aligned}
& =-\frac{f_{B}\left(\xi_{2 b}\right)-f_{B}\left(-\xi_{1^{\prime}+2^{\prime}-2, a}\right)}{\xi_{1^{\prime}+2^{\prime}-2, a}+\xi_{2 b}-i \omega_{n_{1^{\prime}}}-i \omega_{n_{2^{\prime}}}} \\
& =-\frac{f_{B}\left(\xi_{2 b}\right)+f_{B}\left(\xi_{1^{\prime}+2^{\prime}-2, a}\right)+1}{\xi_{1^{\prime}+2^{\prime}-2, a}+\xi_{2 b}-i \omega_{n_{1^{\prime}}}-i \omega_{n_{2^{\prime}}}},
\end{aligned}
$$

where we apply $f_{B}\left(i \omega_{n_{1}}+i \omega_{n_{2}}-\xi\right)=\frac{1}{\exp \left[2 \pi i\left(n_{1}+n_{2}\right)-\beta \xi\right]-1}=$ $\frac{1}{\exp [-\beta \xi]-1}=f_{B}(-\xi)=\frac{1}{e^{-\beta \xi}-1}=-1-\frac{1}{e^{\beta \xi}-1}=-1-f_{B}(\xi)$. Replacing the sum in (21) with (22), $\left\langle S_{\text {int }}^{2}\right\rangle_{>}^{(1)}$ can be rewritten as

$$
\begin{aligned}
\left\langle S_{\mathrm{int}}^{2}\right\rangle_{>}^{(1)}= & \frac{1}{\beta} \sum_{a \neq b} \sum_{3,4} \sum_{1^{\prime}, 2^{\prime}} \mathcal{K}^{2}\left(k_{3}^{a}+k_{4}^{b}\right)\left(k_{2^{\prime}}^{a}+k_{1^{\prime}}^{b}\right) \\
& \times \phi_{1^{\prime} a}^{*} \phi_{2^{\prime} b}^{*} \phi_{3 b} \phi_{4 a} \delta\left(1^{\prime}+2^{\prime}-3-4\right) \\
& \times \int \frac{d^{2} k_{2}}{(2 \pi)^{2}} \frac{f_{B}\left(\xi_{2 b}\right)+f_{B}\left(\xi_{1^{\prime}+2^{\prime}-2, a}\right)+1}{\xi_{1^{\prime}+2^{\prime}-2, a}+\xi_{2 b}-i \omega_{n_{1^{\prime}}}-i \omega_{n_{2^{\prime}}}} \\
& \times\left(k_{2}^{a}-k_{2}^{b}+k_{1^{\prime}}^{b}+k_{2^{\prime}}^{b}\right)^{2} \\
\approx & \frac{1}{\beta} \sum_{a \neq b} \sum_{3,4} \sum_{1^{\prime}, 2^{\prime}} \mathcal{K}^{2}\left(k_{3}^{a}+k_{4}^{b}\right)\left(k_{2^{\prime}}^{a^{\prime}}+k_{1^{\prime}}^{b^{\prime}}\right) \\
& \times \phi_{1^{\prime} a}^{*} \phi_{2^{\prime} b}^{*} \phi_{3 b} \phi_{4 a} \delta\left(1^{\prime}+2^{\prime}-3-4\right) \\
& \times \int \frac{d^{2} k_{2}}{(2 \pi)^{2}} \frac{f_{B}\left(\xi_{2 b}\right)+f_{B}\left(\xi_{2 a}\right)+1}{\xi_{2 a}+\xi_{2 b}-i \omega_{n_{1^{\prime}}}-i \omega_{n_{2^{\prime}}}}\left(k_{2}^{a}-k_{2}^{b}\right)^{2},
\end{aligned}
$$

where the momenta $\mathbf{k}_{\mathbf{1}^{\prime}}$ and $\mathbf{k}_{\mathbf{2}^{\prime}}$ can be ignored in the expression since $\phi_{1^{\prime} a}$ and $\phi_{2^{\prime} b}$ are both slow modes. The integrated region of the momentum $\mathbf{k}_{2}$ in $\left\langle S_{\text {int }}^{2}\right\rangle_{>}^{(1)}$ is given by $(\Lambda / s)^{2} \leqslant$ $\sum_{a(a \neq b)}\left(k_{2}^{b}\right)^{2}+\frac{\mathcal{T}_{1}}{\mathcal{T}_{0}}\left(k_{2}^{a}\right)^{2} \leqslant \Lambda^{2}$ since the momentum $\mathbf{k}_{\mathbf{2}}$ is carried by the field $\phi_{2 b}^{*}$. With substitutions $\tilde{k}^{b}=k_{2}^{b}, \tilde{k}^{a}=\sqrt{\frac{\mathcal{T}_{1}}{\mathcal{T}_{0}}} k_{2}^{a}$, we limit our integrated region to $(\Lambda / s)^{2} \leqslant \tilde{\mathbf{k}}^{2} \leqslant \Lambda^{2}$. Therefore, the contribution from the first type of contraction is given 
by

$$
\begin{aligned}
\left\langle S_{\text {int }}^{2}\right\rangle_{>}^{(1)}= & \frac{A}{\beta} \sum_{a \neq b} \sum_{1^{\prime} 2^{\prime} 34} \mathcal{K}^{2}\left(k_{3}^{a}+k_{4}^{b}\right)\left(k_{2^{\prime}}^{a}+k_{1^{\prime}}^{b}\right) \\
& \times \phi_{1^{\prime} a}^{*} \phi_{2^{\prime} b}^{*} \phi_{3 b} \phi_{4 a} \delta\left(1^{\prime}+2^{\prime}-3-4\right) d l,
\end{aligned}
$$

where the parameter $A$ takes the form of

$$
\begin{aligned}
A & =\frac{1}{d l} \int_{>} \frac{d^{2} k_{2}}{(2 \pi)^{2}} \frac{f_{B}\left(\xi_{2 a}\right)+f_{B}\left(\xi_{2 b}\right)+1}{\xi_{2 a}+\xi_{2 b}-\omega_{1^{\prime}}-\omega_{2^{\prime}}-i 0^{+}}\left(k_{2}^{a}-k_{2}^{b}\right)^{2} \\
& \approx \frac{1}{d l} \int_{>} \frac{d^{2} k_{2}}{(2 \pi)^{2}} \frac{f_{B}\left(\xi_{2 a}\right)+f_{B}\left(\xi_{2 b}\right)+1}{\xi_{2 a}+\xi_{2 b}}\left(k_{2}^{a}-k_{2}^{b}\right)^{2}
\end{aligned}
$$

where we use analytic continuation here and replace the imaginary frequencies with $\omega_{1^{\prime}}$ and $\omega_{2^{\prime}}$. It is necessarily assumed that the frequencies and momenta on the external lines can be ignored compared with $\xi_{0 \Lambda}$ and $\Lambda$. It is obvious that the expression (24) is positive. In other words, $A>0$. More calculations on $A$ are shown in Appendix B. By comparing the form of (23) with the bare interaction vertex,we find that it will correct the interaction parameter $\mathcal{K}$ effectively. According to the effective action $S_{\text {eff }}=S_{0}+\left\langle S_{\text {int }}\right\rangle-\frac{1}{2}\left(\left\langle S_{\text {int }}^{2}\right\rangle-\left\langle S_{\text {int }}\right\rangle^{2}\right)$, we have the form of the $\beta$ function of parameter $\mathcal{K}$ :

$$
\frac{d \mathcal{K}}{d l}=-\mathcal{K}^{2} \times A-2 \mathcal{K},
$$

where the term $-2 \mathcal{K}$ originates from the rescaling of slow momenta carried by the slow modes in (9) above. We have the general forms of $\mathcal{K}(l)$ and $\mathcal{T}_{1}(l)$ :

$$
\begin{gathered}
\mathcal{K}(l)=\frac{2 / A}{\frac{\mathcal{K}(0)+2 / A}{\mathcal{K}(0)} e^{2 l}-1}, \\
\mathcal{T}_{1}(l)=\left[\mathcal{T}_{1}(0)^{3 / 2}+\sqrt{\mathcal{T}_{0}} \frac{3 \Lambda^{2}}{4 A \pi} f_{B}\left(\xi_{0 \Lambda}\right)\right. \\
\left.\times \ln \left(\frac{2}{-\mathcal{K}(0) A e^{-2 l}+[2+\mathcal{K}(0) A]}\right)\right]^{2 / 3},
\end{gathered}
$$

with the separatrix

$$
\mathcal{T}_{1}(0)=\left[f_{B}\left(\xi_{0 \Lambda}\right) \sqrt{\mathcal{T}_{0}} \frac{3 \Lambda^{2}}{4 A \pi} \ln \left(\frac{2+\mathcal{K}(0) A}{2}\right)\right]^{2 / 3} .
$$

The second type of contraction $\left\langle S_{\text {int }}^{2}\right\rangle_{\rangle}^{(2)}$ contains overall eight cases presented below.

Case 5: The momenta $\mathbf{k}_{\mathbf{1}}, \mathbf{k}_{\mathbf{3}}, \mathbf{k}_{\mathbf{2}^{\prime}}$, and $\mathbf{k}_{\mathbf{4}^{\prime}}$ are fast momenta. $\mathbf{k}_{\mathbf{1}}=\mathbf{k}_{\mathbf{4}^{\prime}}$ and $\omega_{n_{1}}=\omega_{n_{4^{\prime}}} ; \mathbf{k}_{\mathbf{3}}=\mathbf{k}_{\mathbf{2}^{\prime}}$ and $\omega_{n_{3}}=\omega_{n_{2^{\prime}}}$.

Case 6: The momenta $\mathbf{k}_{\mathbf{1}}, \mathbf{k}_{\mathbf{4}}, \mathbf{k}_{\mathbf{1}^{\prime}}$, and $\mathbf{k}_{\mathbf{4}^{\prime}}$ are fast momenta. $\mathbf{k}_{\mathbf{1}}=\mathbf{k}_{\mathbf{4}^{\prime}}$ and $\omega_{n_{1}}=\omega_{n_{4^{\prime}}} ; \mathbf{k}_{\mathbf{4}}=\mathbf{k}_{\mathbf{1}^{\prime}}$ and $\omega_{n_{4}}=\omega_{n_{1^{\prime}}}$.

Case 7: The momenta $\mathbf{k}_{\mathbf{1}}, \mathbf{k}_{\mathbf{3}}, \mathbf{k}_{\mathbf{1}^{\prime}}$, and $\mathbf{k}_{\mathbf{3}^{\prime}}$ are fast momenta. $\mathbf{k}_{\mathbf{1}}=\mathbf{k}_{\mathbf{3}^{\prime}}$ and $\omega_{n_{1}}=\omega_{n_{3^{\prime}}} ; \mathbf{k}_{\mathbf{3}}=\mathbf{k}_{\mathbf{1}^{\prime}}$ and $\omega_{n_{3}}=\omega_{n_{1^{\prime}}}$.

Case 8: The momenta $\mathbf{k}_{\mathbf{1}}, \mathbf{k}_{\mathbf{4}}, \mathbf{k}_{\mathbf{2}^{\prime}}$, and $\mathbf{k}_{\mathbf{3}^{\prime}}$ are fast momenta. $\mathbf{k}_{\mathbf{1}}=\mathbf{k}_{\mathbf{3}^{\prime}}$ and $\omega_{n_{1}}=\omega_{n_{3^{\prime}}} ; \mathbf{k}_{\mathbf{4}}=\mathbf{k}_{\mathbf{2}^{\prime}}$ and $\omega_{n_{4}}=\omega_{n_{2^{\prime}}}$.

Case 9: The momenta $\mathbf{k}_{\mathbf{2}}, \mathbf{k}_{\mathbf{3}}, \mathbf{k}_{\mathbf{2}^{\prime}}$, and $\mathbf{k}_{\mathbf{3}^{\prime}}$ are fast momenta. $\mathbf{k}_{\mathbf{2}}=\mathbf{k}_{\mathbf{3}^{\prime}}$ and $\omega_{n_{2}}=\omega_{n_{3^{\prime}}} ; \mathbf{k}_{\mathbf{3}}=\mathbf{k}_{\mathbf{2}^{\prime}}$ and $\omega_{n_{3}}=\omega_{n_{2^{\prime}}}$.

Case 10: The momenta $\mathbf{k}_{\mathbf{2}}, \mathbf{k}_{\mathbf{4}}, \mathbf{k}_{\mathbf{1}^{\prime}}$, and $\mathbf{k}_{\mathbf{3}^{\prime}}$ are fast momenta. $\mathbf{k}_{2}=\mathbf{k}_{\mathbf{3}^{\prime}}$ and $\omega_{n_{2}}=\omega_{n_{3^{\prime}}} ; \mathbf{k}_{\mathbf{4}}=\mathbf{k}_{\mathbf{1}^{\prime}}$ and $\omega_{n_{4}}=\omega_{n_{1^{\prime}}}$.

Case 11: The momenta $\mathbf{k}_{2}, \mathbf{k}_{\mathbf{3}}, \mathbf{k}_{\mathbf{1}^{\prime}}$, and $\mathbf{k}_{\mathbf{4}^{\prime}}$ are fast momenta. $\mathbf{k}_{\mathbf{2}}=\mathbf{k}_{\mathbf{4}^{\prime}}$ and $\omega_{n_{2}}=\omega_{n_{4^{\prime}}} ; \mathbf{k}_{\mathbf{3}}=\mathbf{k}_{\mathbf{1}^{\prime}}$ and $\omega_{n_{3}}=\omega_{n_{1^{\prime}}}$.

Case 12: The momenta $\mathbf{k}_{\mathbf{2}}, \mathbf{k}_{\mathbf{4}}, \mathbf{k}_{\mathbf{2}^{\prime}}$, and $\mathbf{k}_{\mathbf{3}^{\prime}}$ are fast momenta. $\mathbf{k}_{\mathbf{2}}=\mathbf{k}_{\mathbf{3}^{\prime}}$ and $\omega_{n_{2}}=\omega_{n_{3^{\prime}}} ; \mathbf{k}_{\mathbf{4}}=\mathbf{k}_{\mathbf{2}^{\prime}}$ and $\omega_{n_{4}}=\omega_{n_{2^{\prime}}}$.
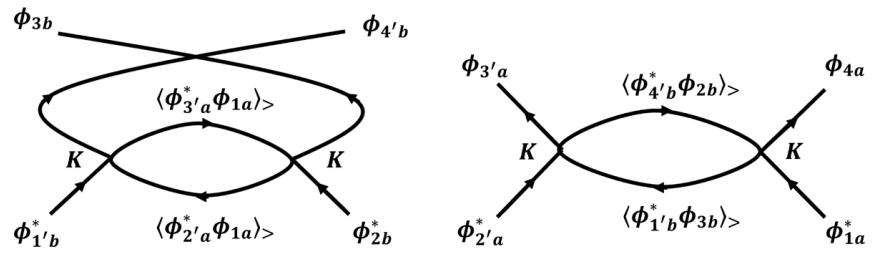

FIG. 3. The Feynman diagrams of the second type of contraction in the vertex correction contribute to the correction of other terms like $g\left|\phi_{a}\right|^{4}$. They correspond to cases 5-12. For simplicity, we here show two of them. By exchanging the symmetric indices, we can have the other six possible diagrams.

The Feynman diagrams related are presented in Fig. 3. After integrating the fast momenta, all of these terms will be turned into terms with constant coefficients. Therefore, integration from the two connected diagrams will be explained as corrections of other possible terms such as $g\left|\phi_{a}\right|^{4}$. We do not care about this parameter because it does not contribute to the parameters $\mathcal{K}$ and $\mathcal{T}_{1}$. For simplicity, we do not present more details here.

\section{Global phase diagram}

In summary, we can see tha the first-order corrections only correct the kinetic terms in directions other than the $a$ th one of field configurations $\phi_{a}$ [see the definition below Eq. (7)]. In this way, the kinetic energy along the ath axis will not be influenced by the contraction. We can directly prove that the higher order correction will still contribute nothing to the parameter $\mathcal{T}_{0}$. Hence, we have the $\beta$ function for parameter $\mathcal{T}_{1}$ corresponding to Fig. 1, given by Eq. (19). We can safely come to the conclusion that if $\mathcal{K}>0$, the parameter $t_{1}$ will reduce in the RG flow. This requires the parameter $\mathcal{K}$ to be positive. Only with positive $\mathcal{K}$ can parameter $\mathcal{T}_{1}$ flow to zero in the RG analysis. Nevertheless, at this step, it is insufficient to tell whether the parameter $\mathcal{T}_{1}$ is irrelevant here since the elements in the matrix $\mathcal{K}$ will also flow to zero. What we need is further calculation on the vertex correction, which has been given in Eq. (25).

A numerical result of $\beta$ functions (19) and (25) is shown in Fig. 4, where the red line is the separatrix (28). In the region below the line, all $\mathcal{T}_{1}(0)$ will flow to zero in RG flow, indicating the emergence of higher rank symmetry in lowenergy physics since those terms violating the higher rank symmetry will vanish in the low-energy physics. In addition, after comparing the parameters $\mathcal{T}_{1}(0)$ and $\mathcal{K}(0) \Lambda^{2}$ near the separatrix, we have $\mathcal{T}_{1}(0) \ll \mathcal{K}(0) \Lambda^{2} \ll \mathcal{T}_{0}$ with the approximation $\frac{\xi_{0} \Lambda}{2 k_{B} T} \gg 1$, which can be realized in cold-atom systems. This tells us our calculation is consistent with our initial assumption above.

From the phase diagram, we can safely come to the conclusion that the higher rank symmetry will emerge after integrating the high-energy modes since off-diagonal kinetic parameters are just perturbations to the system. However, it should be noted that our RG analysis is only valid near the initial point $l=0$. We here also do not take higher order terms into account. Therefore, our calculation and results may be invalidated when $l \rightarrow \infty$. The reason we do not just finish our $\mathrm{RG}$ analysis on the axis $\mathcal{T}_{1}(0)=0$ is that we only consider the 


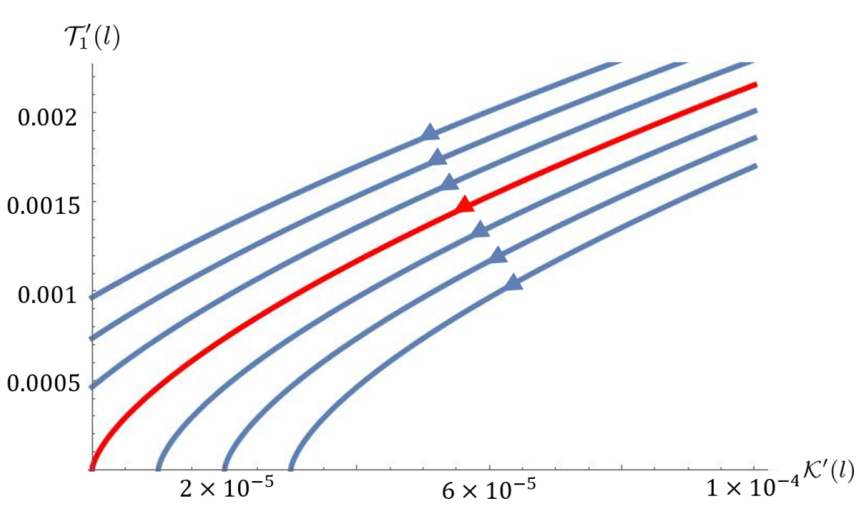

FIG. 4. RG flow for $\mathcal{T}_{1}^{\prime}(l)=\mathcal{T}_{1}(l)\left(f_{B}\left(\xi_{0 \Lambda}\right) \sqrt{\mathcal{T}_{0}} \frac{3 \Lambda^{2}}{4 A \pi}\right)^{-2 / 3}$ and $\mathcal{K}^{\prime}(l)=A \mathcal{K}(l) / 2$. Here the red line is a separatrix below which higher rank symmetry emerges. The parameters $\left(\mathcal{T}_{1}^{\prime}(0), \mathcal{K}^{\prime}(0)\right)$ of these lines are (from top to bottom) $\left(2.57 \times 10^{-3}, 10^{-4}\right),(2.43 \times$ $\left.10^{-3}, 10^{-4}\right), \quad\left(2.30 \times 10^{-3}, 10^{-4}\right), \quad\left(2.16 \times 10^{-3}, 10^{-4}\right), \quad(2.01 \times$ $\left.10^{-3}, 10^{-4}\right),\left(1.86 \times 10^{-3}, 10^{-4}\right)$, and $\left(1.70 \times 10^{-3}, 10^{-4}\right)$.

lower-order corrections. If we start with an initial point on the horizontal axis, $\mathcal{T}_{1}(0)=0$, we have $S_{2} \rightarrow \infty$ in $\frac{d \mathcal{T}_{1}}{d l}$, which requires more higher order terms since they contain expressions with higher order of $S_{2}$. We have to take all of them into account for accuracy, which is beyond the current perturbation calculations. Although our calculation cannot take all the higher order corrections into account, it also exhibits the tendency of RG flow to give an accurate prediction near the region with $l=0$. Alternatively, the exact flat "band" along one direction for a boson, in analogy to bosonic Landau level, leads to strong correlation effect even though interaction is not large. We also comment that the perturbation calculations fail completely if we instead study models [65] with conserved dipole moments, where bosons are fractons that are immobile in all directions. In such models, the Hamiltonian is intrinsically non-Gaussian with no kinetic terms at all. But analytic difficulty becomes much smaller when fracton condensation is considered, as in Ref. [65].

The above discussion is all about the $\beta$ functions of parameters of two-component bosons in two-dimensional space. Similarly, we can extend the case to the $d$-dimensional space with $d>2$. We here give the forms of $\beta$ functions of $d$ component bosonic systems for reference. The $\beta$ functions of $t_{a b}(a \neq b)$ and $K_{a b}(a \neq b)$ are given by

$$
\frac{d t_{a b}}{d l}=-S_{d} K_{a b} f_{B}\left(\xi_{0 \Lambda}\right) \frac{\Lambda^{d}}{(2 \pi)^{d}}, \quad \frac{d K_{a b}}{d l}=-A_{d} K_{a b}^{2}-d K_{a b},
$$

where $S_{d}$ represents the volume of a $d$-dimensional ellipsoid,

$$
\sum_{b=1}^{d} \frac{t_{a b}}{t_{a}}\left(k^{b}\right)^{2}=1,
$$

and

$$
A_{d}=\frac{1}{2 \beta l} \int_{P_{d}} \frac{d^{d} k}{(2 \pi)^{d}} \frac{f_{B}\left(\xi_{k a}\right)+f_{B}\left(\xi_{k b}\right)+1}{\xi_{k a}+\xi_{k b}}\left(k^{a}-k^{b}\right)^{2}
$$

with integrated domain $P_{d}:\left(\Lambda e^{-l}\right)^{2} \leqslant \sum_{b=1}^{d} \frac{t_{a b}}{t_{a}}\left(k^{b}\right)^{2} \leqslant \Lambda^{2}$. They give the same type of RG flow phase diagram as Fig. 4.

\section{SUMMARY AND OUTLOOK}

In this paper, we study how higher rank symmetry (5) and angular moment conservation (6) emerge at low energies through a RG analysis. In other words, we identify them as emergent phenomena rather than strict properties of microscopic models. A phase diagram is given in Fig. 4, in which a wide parameter region is found to support emergent phenomena. Despite the limitation of one-loop perturbation techniques, we argue that emergence occurs in the deep infrared regime. On the other hand, by regarding higher rank symmetry as emergent symmetry, our work opens a door to a new way of thinking on realization of such unconventional symmetry and higher moment conservation in more realistic models, e.g., in simple frustrated spin models near symmetric points. Recently, some higher moment conserving 1D spin systems have been found to support anomalously slow, subdiffusive late-time transport [70,74]. Thus, it is interesting to ask how emergent conservation of angular moments affects the late-time transport. Besides, the Hamiltonian can be reformulated on a square lattice. In an optical lattice of cold-atom experiments, one may choose lattice constant $l=700 \mathrm{~nm}$, $\Lambda \sim \frac{1}{l}=1.43 \times 10^{7} \mathrm{~m}^{-1}, m_{0}=\frac{1}{t_{0}}=8.22 \times 10^{-34} \mathrm{~kg}, T=$ $10 \mathrm{~K}$. The condition $\frac{t_{0} \Lambda^{2}-2 \mu}{2 k_{B} T} \sim 10^{3}$, where the chemical potential is negative indicates the possibility of simulating the two-component bosons on the optical lattice. For accuracy and extension, it will be interesting to further consider the correction from higher order terms with more loops in the Feynman diagram. Finally, it will be interesting to study symmetry-protected topological phases (SPTs) with such emergent higher rank symmetry.

\section{ACKNOWLEDGMENTS}

Discussions with Ruizhi Liu and Yixin Xu are acknowledged. This work of both authors was done in Guangzhou South Campus of Sun Yat-sen University (SYSU) with full financial support from the SYSU talent plan, Guangdong Basic and Applied Basic Research Foundation under Grant No. 2020B1515120100 and National Natural Science Foundation of China Grants No. 11847608 and No. 12074438.

\section{APPENDIX A: HOW THE ANGULAR MOMENT CONSERVATION AFFECTS SINGLE-PARTICLE MOTION}

In the main text, we introduced the following conserved quantities $(a, b=1,2, \ldots, d ; a<b)$ in $d$-dimensional space:

$$
\mathcal{Q}^{a}=\int d^{d} x \rho^{a}, \quad \mathcal{Q}^{a b}=\int d^{d} x\left(\rho^{a} x^{b}-\rho^{b} x^{a}\right)
$$

For each $a$, the conservation of $\mathcal{Q}^{a}$ requires that all $a$ th component bosons are always in the $d$-dimensional space. The conserved quantities $\mathcal{Q}^{a b}$ leads to the mobility restriction that a single $a$ th component boson is only allowed to move along $a$ th directions. To be more specific, we consider $d=2$ and the following three quantities are conserved:

$$
\begin{aligned}
\mathcal{Q}^{1} & =\int d^{d} x \rho^{1}, \quad \mathcal{Q}^{2}=\int d^{d} x \rho^{2}, \\
\mathcal{Q}^{12} & =\int d^{d} x\left(\rho^{1} x^{2}-\rho^{2} x^{1}\right) .
\end{aligned}
$$


$\mathcal{Q}^{1}$ and $\mathcal{Q}^{2}$ are the usual conserved charge (particle number) of bosons of the first and second components, respectively. The conserved quantity $\mathcal{Q}^{12}$ is the total angular moment formed by bosons. Suppose $\mathcal{Q}^{1}=N_{1}$ and $\mathcal{Q}^{2}=N_{2}$. We can use Dirac function to express $\rho^{1}$ and $\rho^{2}$ :

$$
\rho^{1}(\mathbf{x})=\sum_{i}^{N_{1}} \delta\left(\mathbf{x}-\mathbf{x}_{i}\right), \quad \rho^{2}(\mathbf{x})=\sum_{j}^{N_{2}} \delta\left(\mathbf{x}-\mathbf{x}_{j}\right) .
$$

It means that bosons of the first (second) component are located in $\mathbf{x}_{i}\left(\mathbf{x}_{j}\right)$ with $i=1,2,3, \ldots, N_{1}\left(j=1,2,3, \ldots, N_{2}\right)$. Then, $\mathcal{Q}^{12}$ is reduced to

$$
\mathcal{Q}^{12}=\sum_{i}^{N_{1}} x_{i}^{2}-\sum_{j}^{N_{2}} x_{j}^{1}
$$

From this expression, one can conclude that, if we move a first-component boson, in order to keep $\mathcal{Q}^{12}$ invariant, the boson is only allowed to be movable in the first direction such that its coordinate $x^{2}$ is unchanged. Of course, we can collectively move bosons of both components, such that the change in $\sum_{i}^{N_{1}} x_{i}^{2}$ can be canceled out by the change in $\sum_{j}^{N_{2}} x_{j}^{1}$ and so $\mathcal{Q}^{12}$ is unaltered. This scenario is beyond the single-particle movement and gives nontrivial effects when intercomponent interaction $(K$ term) is involved.

\section{APPENDIX B: DETAILS OF THE PARAMETER $A$}

Here we focus on the specific value of the parameter $A$ by taking some approximations. By referencing the definition of $\tilde{\mathbf{k}}$ above, we have

$$
\begin{aligned}
A & =\frac{1}{d l} \int_{>} \frac{d^{2} \tilde{\mathbf{k}}}{(2 \pi)^{2}} \frac{f_{B}\left(\xi_{2 a}\right)+f_{B}\left(\xi_{2 b}\right)+1}{\xi_{2 a}+\xi_{2 b}}\left(k_{2}^{a}-k_{2}^{b}\right)^{2} \sqrt{\frac{\mathcal{T}_{0}}{\mathcal{T}_{1}}} \\
& =\frac{1}{d l} \int_{>} \frac{|\tilde{\mathbf{k}}| d|\tilde{\mathbf{k}}| d \theta}{(2 \pi)^{2}} \frac{f_{B}\left(\xi_{2 a}\right)+f_{B}\left(\xi_{2 b}\right)+1}{\xi_{2 a}+\xi_{2 b}}\left(k_{2}^{a}-k_{2}^{b}\right)^{2} \sqrt{\frac{\mathcal{T}_{0}}{\mathcal{T}_{1}}} \\
& =\frac{1}{d l} \int_{>} \frac{d|\tilde{\mathbf{k}}|^{2} d \theta}{2(2 \pi)^{2}} \frac{f_{B}\left(\xi_{2 a}\right)+f_{B}\left(\xi_{2 b}\right)+1}{\xi_{2 a}+\xi_{2 b}}\left(k_{2}^{a}-k_{2}^{b}\right)^{2} \sqrt{\frac{\mathcal{T}_{0}}{\mathcal{T}_{1}}}
\end{aligned}
$$

For simplicity, we will approximately use $\mathcal{T}_{1}(0)$ to replace $\mathcal{T}_{1}$ below for convenience. Besides, we have $k_{2}^{b}=\Lambda \sin \theta$ and $k_{2}^{a}=\Lambda \sqrt{\frac{\mathcal{T}_{0}}{\mathcal{T}_{1}}} \cos \theta$ in the integrated domain. Hence, the kinetic energies $\xi_{2 a}$ and $\xi_{2 b}$ can be approximately considered as $\xi_{1 \Lambda}=$ $\xi_{0 \Lambda}\left(\frac{\mathcal{T}_{0}}{\mathcal{T}_{1}} \cos ^{2} \theta+\frac{\mathcal{T}_{1}}{\mathcal{T}_{0}} \sin ^{2} \theta\right)$ and $\xi_{0 \Lambda}$ separately if we assume the chemical potential is small enough: $|\mu| \ll \frac{t_{0} \Lambda^{2}}{2}$. In this way, the parameter $A$ can be rewritten as

$$
\begin{aligned}
& A \approx \frac{1}{2 d l} \int_{0}^{2 \pi} \frac{d \theta}{(2 \pi)^{2}}\left(\Lambda^{2}-\left(\frac{\Lambda}{s}\right)^{2}\right) \frac{f_{B}\left(\xi_{1 \Lambda}\right)+f_{B}\left(\xi_{0 \Lambda}\right)+1}{\xi_{1 \Lambda}+\xi_{0 \Lambda}}\left(\Lambda \sqrt{\frac{\mathcal{T}_{0}}{\mathcal{T}_{1}}} \cos \theta-\Lambda \sin \theta\right)^{2} \sqrt{\frac{\mathcal{T}_{0}}{\mathcal{T}_{1}(0)}} \\
& =\Lambda^{4} \int_{0}^{2 \pi} \frac{d \theta}{(2 \pi)^{2}} \frac{f_{B}\left(\xi_{1 \Lambda}\right)+f_{B}\left(\xi_{0 \Lambda}\right)+1}{\xi_{1 \Lambda}+\xi_{0 \Lambda}}\left(\sqrt{\frac{\mathcal{T}_{0}}{\mathcal{T}_{1}}} \cos \theta-\sin \theta\right)^{2} \sqrt{\frac{\mathcal{T}_{0}}{\mathcal{T}_{1}(0)}} \\
& =\Lambda^{4} \int_{0}^{2 \pi} \frac{d \theta}{(2 \pi)^{2}} \frac{f_{B}\left(\xi_{1 \Lambda}\right)+f_{B}\left(\xi_{0 \Lambda}\right)+1}{\xi_{1 \Lambda}+\xi_{0 \Lambda}}\left(\sqrt{\frac{\mathcal{T}_{0}}{\mathcal{T}_{1}}} \cos \theta-\sin \theta\right)^{2} \sqrt{\frac{\mathcal{T}_{0}}{\mathcal{T}_{1}(0)}} \\
& \approx \Lambda^{4} \int_{0}^{2 \pi} \frac{d \theta}{(2 \pi)^{2}} \frac{1}{\left(\xi_{0 \Lambda}+\xi_{1 \Lambda}\right)\left(e^{\xi_{0 \Lambda}\left(\frac{\mathcal{T}_{0}}{\mathcal{T}_{1}} \cos ^{2} \theta+\frac{\mathcal{T}_{1}}{\mathcal{T}_{0}} \sin ^{2} \theta\right) / k_{B} T}-1\right)} \sqrt{\frac{\mathcal{T}_{0}}{\mathcal{T}_{1}(0)}} \\
& +\Lambda \sqrt{\frac{\mathcal{T}_{0}}{\mathcal{T}_{1}(0)}} \int_{0}^{2 \pi} \frac{d \theta}{(2 \pi)^{2}} \frac{f_{B}\left(\xi_{0 \Lambda}\right)+1}{\xi_{1 \Lambda}+\xi_{0 \Lambda}}\left(\sqrt{\frac{\mathcal{T}_{0}}{\mathcal{T}_{1}}} \cos \theta-\sin \theta\right)^{2} \\
& \approx \frac{4 \Lambda^{2}}{\mathcal{T}_{0}} \int_{\frac{\pi}{2}-\mathcal{T}_{1}^{\prime}}^{\frac{\pi}{2}+\mathcal{T}_{1}^{\prime}} \frac{d \theta}{(2 \pi)^{2}} \frac{2 k_{B} T}{\mathcal{T}_{0} \Lambda^{2} \mathcal{T}_{1}^{\prime} \sin ^{2} \theta} \sqrt{\frac{\mathcal{T}_{0}}{\mathcal{T}_{1}(0)}}+\Lambda^{4} \sqrt{\frac{\mathcal{T}_{0}}{\mathcal{T}_{1}(0)}} \int_{0}^{2 \pi} \frac{d \theta}{(2 \pi)^{2}} \frac{\left(\sqrt{\frac{\mathcal{T}_{0}}{\mathcal{T}_{1}}} \cos \theta-\sin \theta\right)^{2}\left[1+f_{B}\left(\xi_{0 \Lambda}\right)\right]}{\xi_{0 \Lambda}\left(1+\frac{\mathcal{T}_{0}}{\mathcal{T}_{1}} \cos ^{2} \theta+\frac{\mathcal{T}_{1}}{\mathcal{T}_{0}} \sin ^{2} \theta\right)},
\end{aligned}
$$

with $\mathcal{T}_{1}^{\prime}=\frac{\mathcal{T}_{1}}{\mathcal{T}_{0}} \ll 1$. Here we approximately consider that $\frac{\mathcal{T}_{1} \Lambda^{2}}{2 k_{B} T} \ll 1$. This expression contains two integrals. The first one includes a trick. Since $\mathcal{T}_{1}^{\prime} \ll 1$, the first integral becomes nontrivial only around the two points, $\theta=\frac{\pi}{2}$ and $\theta=\frac{3 \pi}{2}$, which give the same value. Therefore, we change our integrated region as $\int_{0}^{2 \pi} \rightarrow 2 \int_{\frac{\pi}{2}-\mathcal{T}_{1}^{\prime}}^{\frac{\pi}{2}+\mathcal{T}_{1}^{\prime}}$. In addition, we have $\xi_{1 \Lambda}=$ $\frac{\mathcal{T}_{1} \Lambda^{2}}{2} \ll \xi_{0 \Lambda}$ at $\theta=\frac{\pi}{2}$ and $\theta=\frac{3 \pi}{2}$. In this way, the first integral given by

$$
\frac{4 \Lambda^{2}}{\mathcal{T}_{0}} \int_{\frac{\pi}{2}-\mathcal{T}_{1}^{\prime}}^{\frac{\pi}{2}+\mathcal{T}_{1}^{\prime}} \frac{d \theta}{(2 \pi)^{2}} \frac{2 k_{B} T}{\mathcal{T}_{0} \Lambda^{2} \mathcal{T}_{1}^{\prime} \sin ^{2} \theta} \sqrt{\frac{\mathcal{T}_{0}}{\mathcal{T}_{1}(0)}}
$$

can be figured out to be

$$
\frac{4 \Lambda^{2}}{\mathcal{T}_{0}} \frac{4 k_{B} T}{(2 \pi)^{2} \mathcal{T}_{0} \Lambda^{2}} \sqrt{\frac{\mathcal{T}_{0}}{\mathcal{T}_{1}(0)}} .
$$


The second one,

$$
\Lambda^{4} \sqrt{\frac{\mathcal{T}_{0}}{\mathcal{T}_{1}(0)}} \int_{0}^{2 \pi} \frac{d \theta}{(2 \pi)^{2}} \frac{\left(\sqrt{\frac{\mathcal{T}_{0}}{\mathcal{T}_{1}}} \cos \theta-\sin \theta\right)^{2}\left[1+f_{B}\left(\xi_{0 \Lambda}\right)\right]}{\xi_{0 \Lambda}\left(1+\frac{\mathcal{T}_{0}}{\mathcal{T}_{1}} \cos ^{2} \theta+\frac{\mathcal{T}_{1}}{\mathcal{T}_{0}} \sin ^{2} \theta\right)}
$$

can be proved to be

$$
\frac{\Lambda^{2}}{\pi \mathcal{T}_{0}} \frac{\frac{\mathcal{T}_{0}}{\mathcal{T}_{1}(0)}}{\frac{\mathcal{T}_{0}}{\mathcal{T}_{1}(0)}+1}\left[1+f_{B}\left(\xi_{0 \Lambda}\right)\right] \sqrt{\frac{\mathcal{T}_{0}}{\mathcal{T}_{1}(0)}} .
$$

[1] D. J. Griffiths, Introduction to Electrodynamics, 4th ed. (Pearson, Boston, 2013), republished by Cambridge University Press in 2017.

[2] R. M. Nandkishore and M. Hermele, Fractons, Annu. Rev. Condens. Matter Phys. 10, 295 (2019).

[3] M. Pretko, X. Chen, and Y. You, Fracton phases of matter, Int. J. Mod. Phys. A 35, 2030003 (2020).

[4] M. Pretko, The fracton gauge principle, Phys. Rev. B 98, 115134 (2018).

[5] M. Pretko, Generalized electromagnetism of subdimensional particles: A spin liquid story, Phys. Rev. B 96, 035119 (2017).

[6] M. Pretko, Subdimensional particle structure of higher rank $U$ (1) spin liquids, Phys. Rev. B 95, 115139 (2017).

[7] A. Gromov, Towards Classification of Fracton Phases: The Multipole Algebra, Phys. Rev. X 9, 031035 (2019).

[8] N. Seiberg and S.-H. Shao, Exotic $U(1)$ symmetries, duality, and fractons in 3+1-dimensional quantum field theory, SciPost Phys. 9, 46 (2020).

[9] S. Vijay, J. Haah, and L. Fu, A new kind of topological quantum order: A dimensional hierarchy of quasiparticles built from stationary excitations, Phys. Rev. B 92, 235136 (2015).

[10] S. Vijay, J. Haah, and L. Fu, Fracton topological order, generalized lattice gauge theory, and duality, Phys. Rev. B 94, 235157 (2016).

[11] A. Prem, J. Haah, and R. Nandkishore, Glassy quantum dynamics in translation invariant fracton models, Phys. Rev. B 95, 155133 (2017).

[12] C. Chamon, Quantum Glassiness in Strongly Correlated Clean Systems: An Example of Topological Overprotection, Phys. Rev. Lett. 94, 040402 (2005).

[13] W. Shirley, K. Slagle, and X. Chen, Foliated fracton order from gauging subsystem symmetries, SciPost Phys. 6, 41 (2019).

[14] H. Ma, E. Lake, X. Chen, and M. Hermele, Fracton topological order via coupled layers, Phys. Rev. B 95, 245126 (2017).

[15] J. Haah, Local stabilizer codes in three dimensions without string logical operators, Phys. Rev. A 83, 042330 (2011).

[16] D. Bulmash and M. Barkeshli, Gauging fractons: Immobile non-Abelian quasiparticles, fractals, and position-dependent degeneracies, Phys. Rev. B 100, 155146 (2019).

[17] A. Prem and D. Williamson, Gauging permutation symmetries as a route to non-Abelian fractons, SciPost Phys. 7, 068 (2019).

[18] D. Bulmash and M. Barkeshli, Generalized $U(1)$ gauge field theories and fractal dynamics, arXiv:1806.01855.

[19] K. T. Tian, E. Samperton, and Z. Wang, Haah codes on general three-manifolds, Ann. Phys. (NY) 412, 168014 (2020).

[20] Y. You, D. Litinski, and F. von Oppen, Higher-order topological superconductors as generators of quantum codes, Phys. Rev. B 100, 054513 (2019).
Combined with these two results, the expression of $A$ is given by

$$
\begin{aligned}
A & =\frac{\Lambda^{2}}{\pi \mathcal{T}_{0}} \sqrt{\frac{\mathcal{T}_{0}}{\mathcal{T}_{1}(0)}}\left(\frac{4 k_{B} T}{\pi \mathcal{T}_{0} \Lambda^{2}}+\frac{\frac{\mathcal{T}_{0}}{\mathcal{T}_{1}}}{\frac{\mathcal{T}_{0}}{\mathcal{T}_{1}}+1}\left[1+f_{B}\left(\xi_{0 \Lambda}\right)\right]\right) \\
& \approx \frac{\Lambda^{2}}{\pi \mathcal{T}_{0}} \sqrt{\frac{\mathcal{T}_{0}}{\mathcal{T}_{1}(0)}}\left(\frac{4 k_{B} T}{\pi \mathcal{T}_{0} \Lambda^{2}}+1+f_{B}\left(\xi_{0 \Lambda}\right)\right)>0,
\end{aligned}
$$

[21] H. Ma, M. Hermele, and X. Chen, Fracton topological order from the Higgs and partial-confinement mechanisms of ranktwo gauge theory, Phys. Rev. B 98, 035111 (2018).

[22] K. Slagle and Y. B. Kim, Fracton topological order from nearest-neighbor two-spin interactions and dualities, Phys. Rev. B 96, 165106 (2017).

[23] G. B. Halász, T. H. Hsieh, and L. Balents, Fracton Topological Phases from Strongly Coupled Spin Chains, Phys. Rev. Lett. 119, 257202 (2017).

[24] K. T. Tian and Z. Wang, Generalized Haah codes and fracton models, arXiv:1902.04543.

[25] W. Shirley, K. Slagle, and X. Chen, Fractional excitations in foliated fracton phases, Ann. Phys. (NY) 410, 167922 (2019).

[26] K. Slagle, D. Aasen, and D. Williamson, Foliated field theory and string-membrane-net condensation picture of fracton order, SciPost Phys. 6, 43 (2019).

[27] W. Shirley, K. Slagle, Z. Wang, and X. Chen, Fracton Models on General Three-Dimensional Manifolds, Phys. Rev. X 8 , 031051 (2018).

[28] A. Prem, S.-J. Huang, H. Song, and M. Hermele, Cage-Net Fracton Models, Phys. Rev. X 9, 021010 (2019).

[29] S. Pai, M. Pretko, and R. M. Nandkishore, Localization in Fractonic Random Circuits, Phys. Rev. X 9, 021003 (2019).

[30] S. Pai and M. Pretko, Dynamical Scar States in Driven Fracton Systems, Phys. Rev. Lett. 123, 136401 (2019).

[31] P. Sala, T. Rakovszky, R. Verresen, M. Knap, and F. Pollmann, Ergodicity Breaking Arising from Hilbert Space Fragmentation in Dipole-Conserving Hamiltonians, Phys. Rev. X 10, 011047 (2020).

[32] A. Kumar and A. C. Potter, Symmetry-enforced fractonicity and two-dimensional quantum crystal melting, Phys. Rev. B 100, 045119 (2019).

[33] L. Radzihovsky and M. Hermele, Fractons from Vector Gauge Theory, Phys. Rev. Lett. 124, 050402 (2020).

[34] A. Dua, I. H. Kim, M. Cheng, and D. J. Williamson, Sorting topological stabilizer models in three dimensions, Phys. Rev. B 100, 155137 (2019).

[35] A. Gromov, Chiral Topological Elasticity and Fracton Order, Phys. Rev. Lett. 122, 076403 (2019).

[36] Y. You, T. Devakul, S. L. Sondhi, and F. J. Burnell, Fractonic Chern-Simons and BF theories, Phys. Rev. Research 2, 023249 (2020).

[37] J. Sous and M. Pretko, Fractons from polarons and hole-doped antiferromagnets: Microscopic realizations, Phys. Rev. B 102 214437 (2020).

[38] V. Khemani, M. Hermele, and R. Nandkishore, Localization from hilbert space shattering: From theory to physical realizations, Phys. Rev. B 101, 174204 (2020). 
[39] J. Wang and K. Xu, Higher-rank tensor field theory of nonAbelian fracton and embeddon, Ann. Phys. 424, 168370 (2021).

[40] J. Wang and S.-T. Yau, Non-Abelian gauged fracton matter field theory: Sigma models, superfluids, and vortices, Phys. Rev. Research 2, 043219 (2020).

[41] S. Pai and M. Pretko, Fractonic line excitations: An inroad from three-dimensional elasticity theory, Phys. Rev. B 97, 235102 (2018).

[42] M. Pretko and R. M. Nandkishore, Localization of extended quantum objects, Phys. Rev. B 98, 134301 (2018).

[43] D. J. Williamson, Z. Bi, and M. Cheng, Fractonic matter in symmetry-enriched $U(1)$ gauge theory, Phys. Rev. B 100, 125150 (2019).

[44] A. Dua, D. J. Williamson, J. Haah, and M. Cheng, Compactifying fracton stabilizer models, Phys. Rev. B 99, 245135 (2019).

[45] B. Shi and Y.-M. Lu, Deciphering the nonlocal entanglement entropy of fracton topological orders, Phys. Rev. B 97, 144106 (2018).

[46] H. Song, A. Prem, S.-J. Huang, and M. A. Martin-Delgado, Twisted fracton models in three dimensions, Phys. Rev. B 99, 155118 (2019).

[47] H. Ma and M. Pretko, Higher-rank deconfined quantum criticality at the Lifshitz transition and the exciton Bose condensate, Phys. Rev. B 98, 125105 (2018).

[48] J. Wang, K. Xu, and S.-T. Yau, Higher-Rank Non-Abelian Tensor Field Theory: Higher-moment or subdimensional polynomial global symmetry, algebraic variety, Noether's theorem, and gauge, Phys. Rev. Research 3, 013185 (2021).

[49] K. Slagle, Foliated quantum field theory of fracton order, Phys. Rev. Lett. 126, 101603 (2021).

[50] D. J. Williamson and T. Devakul, Type-II fractons from coupled spin chains and layers, Phys. Rev. B 103, 155140 (2021).

[51] P. Gorantla, H. Tat Lam, N. Seiberg, and S.-H. Shao, More exotic field theories in 3+1 dimensions, SciPost Phys. 9, 073 (2020).

[52] D. X. Nguyen, A. Gromov, and S. Moroz, Fracton-elasticity duality of two-dimensional superfluid vortex crystals: defect interactions and quantum melting, SciPost Phys. 9, 076 (2020).

[53] M. Pretko, S. A. Parameswaran, and M. Hermele, Odd fracton theories, proximate orders, and parton constructions, Phys. Rev. B 102, 205106 (2020).

[54] D. J. Williamson and M. Cheng, Designer non-Abelian fractons from topological layers, arXiv:2004.07251.

[55] N. Seiberg and S.-H. Shao, Exotic $\mathbb{Z}_{N}$ Symmetries, duality, and fractons in 3+1-dimensional quantum field theory, SciPost Phys. 10, 003 (2021).
[56] D. T. Stephen, J. Garre-Rubio, A. Dua, and D. J. Williamson, Subsystem symmetry enriched topological order in three dimensions, Phys. Rev. Res. 2, 033331 (2020).

[57] A. Gromov, A. Lucas, and R. M. Nandkishore, Fracton hydrodynamics, Phys. Rev. Res. 2, 033124 (2020).

[58] J. Wang, Non-liquid cellular states, arXiv:2002.12932.

[59] W. Shirley, Fractonic order and emergent fermionic gauge theory, arXiv:2002.12026 [cond-mat.str-el].

[60] D. Aasen, D. Bulmash, A. Prem, K. Slagle, and D. J. Williamson, Topological defect networks for fractons of all types, Phys. Rev. Research 2, 043165 (2020).

[61] X.-G. Wen, A systematic construction of gapped non-liquid states, Phys. Rev. Research 2, 033300 (2020).

[62] T. F. J. Poon and X.-J. Liu, Quantum phase transition of fracton topological orders, Phys. Rev. Research 3, 043114 (2021).

[63] M.-Y. Li and P. Ye, Fracton physics of spatially extended excitations, Phys. Rev. B 101, 245134 (2020).

[64] M.-Y. Li and P. Ye, Fracton physics of spatially extended excitations. II. Polynomial ground state degeneracy of exactly solvable models, arXiv:2104.05735.

[65] J.-K. Yuan, S. A. Chen, and P. Ye, Fractonic superfluids, Phys. Rev. Research 2, 023267 (2020).

[66] S. A. Chen, J.-K. Yuan, and P. Ye, Fractonic superfluids. II. Condensing subdimensional particles, Phys. Rev. Res. 3, 013226 (2021).

[67] S. Moudgalya, B. A. Bernevig, and N. Regnault, Quantum many-body scars in a landau level on a thin torus, Phys. Rev. B 102, 195150 (2020).

[68] S. R. Taylor, M. Schulz, F. Pollmann, and R. Moessner, Experimental probes of stark many-body localization, Phys. Rev. B 102, 054206 (2020).

[69] T. Rakovszky, P. Sala, R. Verresen, M. Knap, and F. Pollmann, Statistical localization: From strong fragmentation to strong edge modes, Phys. Rev. B 101, 125126 (2020).

[70] J. Feldmeier, P. Sala, G. De Tomasi, F. Pollmann, and M. Knap, Anomalous Diffusion in Dipole- and Higher-MomentConserving Systems, Phys. Rev. Lett. 125, 245303 (2020).

[71] H. Yan, O. Benton, L. D. C. Jaubert, and N. Shannon, Rank-2 $U$ (1) Spin Liquid on the Breathing Pyrochlore Lattice, Phys. Rev. Lett. 124, 127203 (2020).

[72] R. Shankar, Renormalization-group approach to interacting fermions, Rev. Mod. Phys. 66, 129 (1994).

[73] Z. Komargodski and A. Schwimmer, On renormalization group flows in four dimensions, J. High Energy Phys. 12 (2011) 099.

[74] S. Moudgalya, A. Prem, D. A. Huse, and A. Chan, Spectral statistics in constrained many-body quantum chaotic systems, Phys. Rev. Research 3, 023176 (2021). 